\title{
X-RAY SPECTRAL VARIABILITY IN NGC 3783
}

\author{
R. C. Reis ${ }^{1,2}$, A. C. Fabian ${ }^{2}$, C. S. Reynolds ${ }^{3,4}$, L. W. Brenneman ${ }^{5}$, D. J. Walton ${ }^{2}$, M. Trippe ${ }^{3,4}$, J. M. Miller ${ }^{1}$, \\ R. F. MushotZKY ${ }^{3,4}$, AND M. A. NowaK ${ }^{6}$ \\ ${ }^{1}$ Department of Astronomy, University of Michigan, Ann Arbor, MI 48109, USA \\ 2 Institute of Astronomy, University of Cambridge, Madingley Road, Cambridge, CB3 0HA, UK \\ ${ }^{3}$ Department of Astronomy, University of Maryland, College Park, MD 20742, USA \\ ${ }^{4}$ Joint Space Science Institute (JSI), University of Maryland, College Park, MD 20742, USA \\ ${ }^{5}$ Harvard-Smithsonian CfA, 60 Garden Street MS-67, Cambridge, MA 02138, USA \\ ${ }^{6}$ MIT Kavli Institute for Astrophysics, Cambridge, MA 02139, USA \\ Received 2011 June 14; accepted 2011 October 24; published 2012 January 3
}

\begin{abstract}
NGC 3783 was observed for approximately $210 \mathrm{ks}$ by Suzaku and in this time showed significant spectral and flux variability at both short $(20 \mathrm{ks})$ and long $(100 \mathrm{ks})$ timescales. The full observation is found to consist of approximately six "spectral periods" where the behavior of the soft $(0.3-1.0 \mathrm{keV})$ and hard $(2-10 \mathrm{keV})$ bands are somewhat distinct. Using a variety of methods we find that the strong warm absorber present in this source does not change on these timescales, confirming that the broadband variability is intrinsic to the central source. The timeresolved difference-spectra are well modeled with an absorbed power law below $10 \mathrm{keV}$, but show an additional hard excess at $\approx 20 \mathrm{keV}$ in the latter stages of the observation. This suggests that, in addition to the variable power law, there is a further variable component that varies with time but not monotonically with flux. We show that a likely interpretation is that this further component is associated with variations in the reflection fraction or possibly ionization state of the accretion disk a few gravitational radii from the black hole.
\end{abstract}

Key words: accretion, accretion disks - black hole physics - galaxies: active - galaxies: individual (NGC 3783)

Online-only material: color figures

\section{INTRODUCTION}

Active galactic nuclei (AGNs) are commonly found to display persistent X-ray spectral and flux variability in a variety of timescales (e.g., McHardy 1989; Green et al. 1993). Studies of this broadband variability usually suggest that at least part of this variability is the result of strong gravitational effects close to the central black hole. Time-resolved spectral analyses of such sources are thus an important tool in the study of the inner regions of the accretion flow around supermassive black holes, providing information on both the geometry and physical evolution of the inner accretion disk and on intrinsic physical parameters such as black hole mass and spin.

It has been shown for a number of Seyfert galaxies, most notably MCG-6-30-15 (see, e.g., Fabian \& Vaughan 2003), that the spectrum above a few $\mathrm{keV}$ becomes harder at lower $\mathrm{X}$-ray fluxes. At all flux levels, however, the X-ray spectrum of such systems usually consists of a primary continuum that is accurately approximated as a power law, together with a broad $\mathrm{Fe} \mathrm{K} \alpha$ line and cold reflection features (Nandra et al. 1997). In some cases it is also accompanied by highly ionized warm absorbers and a "soft excess" (Halpern 1984; Reynolds 1997). The most common interpretation for the spectra of such AGNs is that the primary X-ray emission irradiates the underlying optically thick material in the innermost part of the accretion disk, resulting in "reflection signatures" consisting of fluorescent and recombination emission lines as well as absorption features. The most prominent signature is the broad, skewed Fe K $\alpha$ line (see, e.g., Miller 2007 for a recent review of relativistically broadened lines). Furthermore, the soft excess is a natural consequence of reflection from the innermost regions around a black hole for a wide range of ionization states.

Recent spectral variability studies of Seyfert galaxies such as MCG-6-30-15 (Miniutti et al. 2007), 1H0707-495 (Fabian et al.
2009; Zoghbi et al. 2010), and NGC 4051 (Uttley et al. 2004; Terashima et al. 2009) have found different explanations for the origin of the variability-sometimes in the same object. Amongst the possibilities are (1) the spectrum consists of a soft component (power law) with constant spectral slope and variable flux, together with a constant hard component associated with strong reflection from the innermost accretion disk (e.g., MCG-6-30-15; Vaughan et al. 2003; Taylor et al. 2003; Miniutti et al. 2007); (2) the soft component consists of a pivoting power law with the photon index increasing with the logarithm of the flux, together with a constant reflection component (e.g., NGC 4051; Uttley et al. 2004); and (3) a pivoting power law together with a constant, partially covered, neutral (cold) reflection (e.g., NGC 4051; Terashima et al. 2009). Suzaku provides an ideal opportunity to discern between these various distinct interpretations due to its broadband spectral coverage and good signal to noise in the full $0.5-50 \mathrm{keV}$ range, as well as large effective area below $\sim 10 \mathrm{keV}$.

NGC 3783 is a bright, nearby Seyfert 1 galaxy exhibiting prominent broad emission lines and strong X-ray absorption features. Based on a total of approximately $900 \mathrm{ks}$ of Chandra observations, various authors concluded that this source contains three zones of ionized absorption covering a large range of ionization (Kaspi et al. 2002; Netzer et al. 2003; Krongold et al. 2003, 2005). Analyses of the grating data showed little, if any, evidence for changes in the optical depths of the absorbers. Similar conclusions have also been made using $X M M$-Newton reflection grating spectrometer data (Blustin et al. 2002). We observed NGC 3783 for approximately $340 \mathrm{ks}$ with Suzaku as part of the Suzaku AGN Spin Survey Key Project. A detailed analysis of the time-averaged spectrum is presented in Brenneman et al. (2011, hereafter Paper I). For the first time in NGC 3783, the dimensionless spin parameter for the central black hole was estimated to be $>0.88$ at the $99 \%$ level 
of confidence. This was based on the self-consistent modeling of the full reflection features, while simultaneously accounting for absorption due to the warm absorber in the line of sight. In this paper we present an examination of the X-ray spectrum and spectral variability of NGC 3783 using this same long Suzaku observation with the goal of better understanding the accretion disk flow geometry and evolution of this system. By usage of a variety of techniques we show that the spectral and flux variation seen in NGC 3783 are likely due to a combination of a varying power law (with constant index) as well as a hardreflection component. Furthermore, we find that the ionization and reflection fraction in the innermost region of the accretion disk are also likely to be varying.

We start in Section 2 with specifics of the observation and data reduction procedure. This is then followed by a detailed analysis of the spectral variability (Section 3) using a combination of flux-flux relations (Section 3.2), flux-resolved (Section 3.3), and time-resolved difference-spectra (Section 3.4). We apply the results of the previous sections to each individual time period in (Section 3.5.1). Our results are then summarized in Section 4.

\section{DATA REDUCTION}

We observed NGC 3783 for a total of $\sim 340 \mathrm{ks}$ (yielding $210 \mathrm{ks}$ of good "on source" exposure) with Suzaku (Mitsuda et al. 2007) as part of the Suzaku AGN Spin Survey Key Project. The observations were performed during the period 2009 July 10-15. The three operating detectors constituting the X-ray imaging spectrometer (XIS; Koyama et al. 2007) were operated in the "normal" clock and the $3 \times 3$ and $5 \times 5$ editing modes. The observation resulted in a total (co-added) exposure of approximately 420 and $210 \mathrm{ks}$ for the front-illuminated (FI) and back-illuminated (BI) instruments, respectively.

Using the latest HEASOFT v6.10 software package we processed the unfiltered event files for each of the XIS CCDs and editing modes operational, following the Suzaku Data Reduction Guide. ${ }^{7}$ We started by re-running the Suzaku pipeline with the latest calibration (2011 February 10), as well as the associated screening criteria files in order to create new cleaned event files. XSELECT was used to extract spectral products from these event files. The good time intervals provided by the XIS team were employed in all cases to exclude telemetry saturations. Source events were extracted from a circular region of 200 pixels $\left(\sim 208^{\prime \prime}\right)$ radius centered on the point source, and background spectra from another region of the same size, devoid of any obvious contaminating emission. The script XISRESP, ${ }^{8}$ which calls the tools XISRMFGEN and XISSIMARFGEN, was used with the "medium" input to obtain individual ancillary response files (arfs) and redistribution matrix files (rmfs). Finally, we combined the spectra and response files from the two FI instruments (XIS0 and XIS3) using the FTOOL ADDASCASPEC to increase signal to noise. The spectra, background, and responses were then rebinned by a factor of eight to 512 channels using RBNPHA and RBNRMF. The FTOOL GRPPHA was used to give at least 100 counts per spectral bin. Throughout this paper BI and FI spectra are fitted in the $0.5-8.0 \mathrm{keV}$ and $0.7-10 \mathrm{keV}$ energy range, respectively. Unless stated otherwise, the $1.7-2.3 \mathrm{keV}$ energy is ignored due to the possible presence of uncalibrated instrumental features. All energy axes are in the observed frame.

\footnotetext{
7 http://heasarc.gsfc.nasa.gov/docs/suzaku/analysis/

8 http://suzaku.gsfc.nasa.gov/docs/suzaku/analysis/xisresp
}

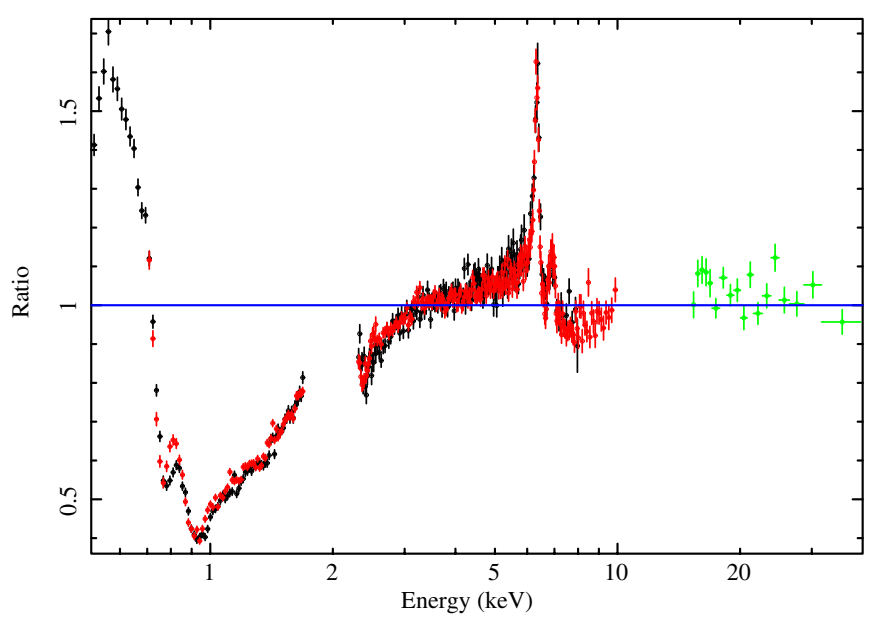

Figure 1. Time-averaged data/model ratio to an absorbed power law. XIS back- and front-illuminated spectra are shown in black and red, respectively. The PIN data are shown in green. A power law was fitted in the 3.0-4.0 and $7.5-40.0 \mathrm{keV}$ energy range. The residuals show the various complexities including the presence of a strong warm absorber seen at soft energies together with a superposition of emission lines at $\sim 6.4 \mathrm{keV}$.

(A color version of this figure is available in the online journal.)

For the hard X-ray detector (HXD; Takahashi et al. 2007) we again reprocessed the unfiltered event files following the data reduction guide (only the PIN data are used in this analyses). Since the HXD is a collimating rather than an imaging instrument, estimating the background requires individual consideration of the non-X-ray instrumental background (NXB) and cosmic X-ray background (CXB). The appropriate response file was downloaded ${ }^{9}$ together with the tuned (Model D) background. A common good-time interval was obtained with MGTIME which combines the good time of the event and background file, and XSELECT was used to extract spectral products. Dead time corrections were applied with HXDDTCOR, and the exposures of the NXB spectra were increased by a factor of 10 , as instructed by the data reduction guide. The contribution from the CXB was simulated using the form of Boldt (1987), with the appropriate normalization for the HXD nominal pointing. The NXB and CXB spectra were then combined using MATHPHA to give a total background spectrum, to which a $2 \%$ systematic uncertainty was added. The PIN spectrum was grouped to have a minimum of 500 counts per energy bin to improve statistics, and again allow the use of $\chi^{2}$ minimization during spectral fitting. The PIN data reduction yielded a total source rate of $(3.27 \pm 0.02) \times 10^{-1}$ count s$^{-1}$ which is $38.5 \%$ of the total observed flux, with a good exposure time of $\approx 234 \mathrm{ks}$. We note here that since the main purpose of our analysis is to investigate the spectral variability of NGC 3783, all PIN spectra presented in this paper are constrained to be strictly simultaneous with their XIS counterpart. Furthermore, we restrict all our PIN analyses to the energy range $15.0-40.0 \mathrm{keV}$ and fit it simultaneously with the XIS data by adding a normalization factor of $1.18^{10}$ with respect to that of the FI spectrum, as recommended in the Suzaku Data Reduction Guide.

\section{DATA ANALYSES AND RESULTS}

Figure 1 shows the time-averaged XIS/PIN spectra for the entire data set compared to a simple absorbed power-law model

\footnotetext{
9 http://www.astro.isas.ac.jp/suzaku/analysis/hxd/

${ }^{10}$ See Paper I for a discussion of the effects in letting this cross normalization vary.
} 


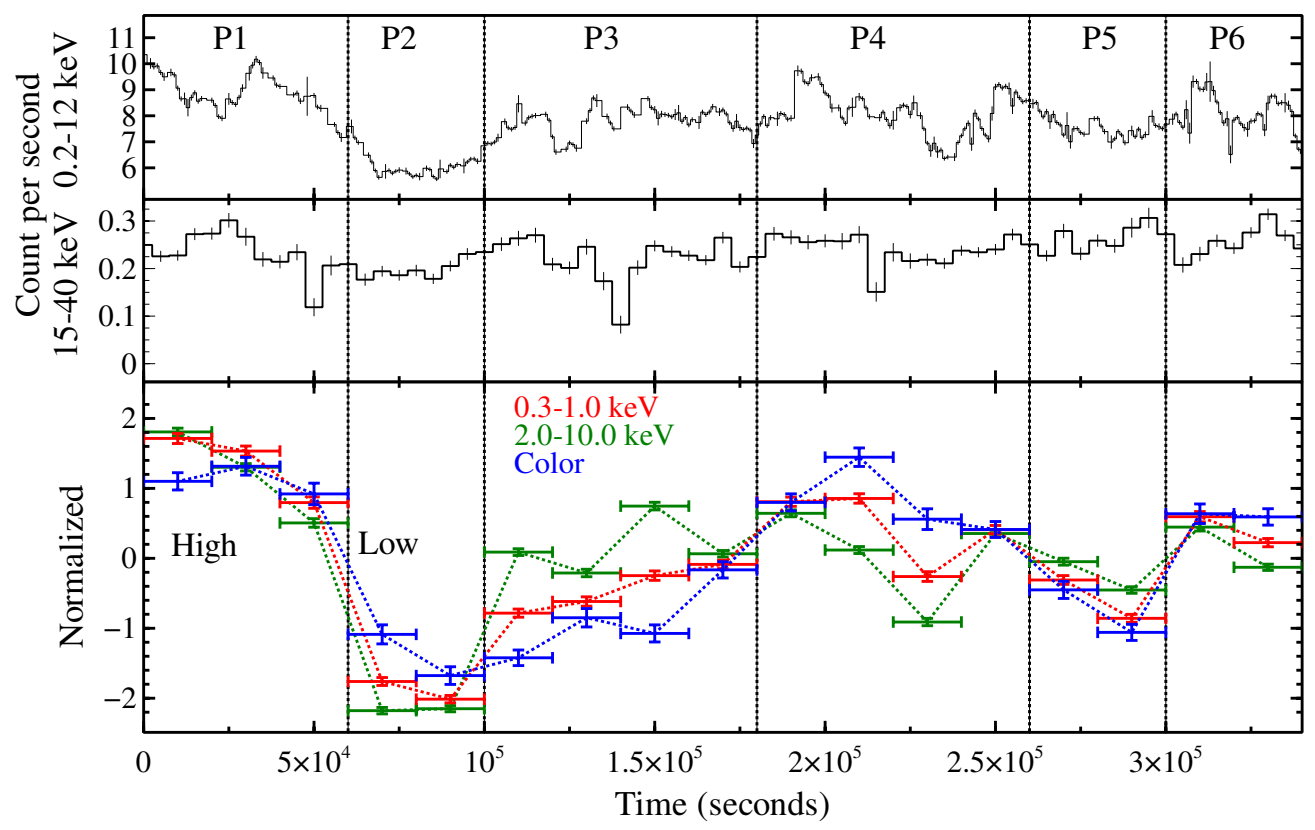

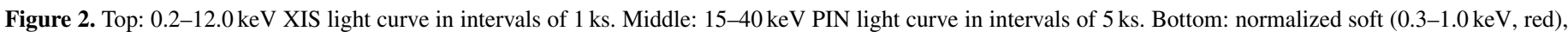

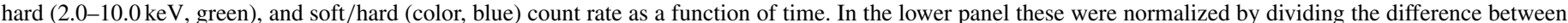

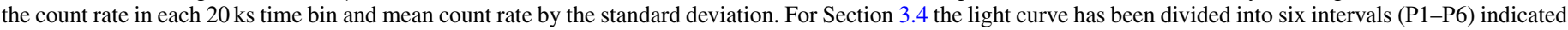
by the dotted vertical lines. We also indicate intervals where both the hard, soft, and softness ratio are at distinct high and low levels.

(A color version of this figure is available in the online journal.)

$\left(N_{\mathrm{H}}\right.$ frozen at the value of $9.91 \times 10^{20} \mathrm{~cm}^{-2}$ as found in Paper I) fitted between $3.0-4.0 \mathrm{keV}$ and $7.5-40.0 \mathrm{keV}$. The presence of a strong warm absorber is clearly seen below $2 \mathrm{keV}$ as is the presence of various emission features peaking at $6.4 \mathrm{keV}$. Detailed fits to the full Suzaku spectrum were presented in Paper I. It was found that the time-averaged spectrum was fitted well by a model consisting of a power-law continuum, blackbody-like soft excess, and X-ray reflection from both cold/neutral distant material and an ionized accretion disk; the observed emission from these components are also strongly affected by a three-zone warm absorber. In order to adequately describe the time-averaged spectrum, a fraction $(\sim 15 \%)$ of the continuum emission scatters around or leaks through the warm absorbing gas. See Paper I (Table 1 therein) for a detailed discussion of the time-average spectral model.

In the following sections we will concentrate on the spectral variation of NGC 3783, initially using a number of modelindependent techniques.

\subsection{Color Changes}

It can be seen from the background-subtracted light curve in Figure 2 (top) that the X-ray continuum emission in NGC 3783 shows clear flux variability on a variety of timescales. The light curve shown above for the XIS data has bin sizes of $1 \mathrm{ks}$ and is the result of the combined data for the three XIS instruments. The PIN light curve in the $15-40 \mathrm{keV}$ range is shown in the middle panel with bin sizes of $5 \mathrm{ks}$. In the bottom panel we show the normalized $0.3-1.0 \mathrm{keV}$ (red; soft) and $2.0-10.0 \mathrm{keV}$ (green; hard) count rate together with the color (blue), defined here as the ratio between soft and hard count rate. In order to better show the evolution of the color, soft, and hard energy band, we have split the data into $20 \mathrm{ks}$ time intervals. During the full observing campaign, NGC 3783 initially dropped in flux by a factor of $\sim 1.8$ in the first $100 \mathrm{ks}$ and subsequently went back to an intermediate flux level during the latter $\sim 200 \mathrm{ks}$. When comparing the evolution of the soft, hard, and softness ratio, we see that there are approximately six distinct periods (P1-P6) which have been highlighted in Figure 2. During the first and last two periods the softness ratio appears to follow both hard and soft normalized rate variation. However, this is not the case in periods 3 and 4 where the softness ratio is significantly below and above the two normalized flux bands, respectively. In order to characterize these spectral variations in a model-independent manner, we begin by applying the flux-flux analysis outlined in Taylor et al. (2003).

\subsection{Flux-Flux Analysis}

Taylor et al. (2003) showed that for Seyfert galaxies, the flux measured in a specific energy band, $F(E)$, can be expressed as the sum of constant $\left(C_{\mathrm{s}}\right.$ and $\left.C_{\mathrm{h}}\right)$ and variable $\left(F_{\mathrm{s}}\right.$ and $\left.F_{\mathrm{h}}\right)$ components, so that the flux in a hard energy band (subscript h) can be related to that of a soft band (subscript s) by the expression

$$
F_{\mathrm{h}}=k\left(F_{\mathrm{s}}-C_{\mathrm{s}}\right)^{\alpha}+C_{\mathrm{h}} .
$$

If the varying component has a constant spectral shape, the flux-flux relation will be linear $(\alpha=1)$. If, however, the spectral variability is intrinsic to a single pivoting power law with no contribution from any other component then plotting $F_{\mathrm{h}}$ versus $F_{\mathrm{s}}$ will result in a simple power law of the form $F_{\mathrm{h}}=k F_{\mathrm{s}}^{\alpha}$ where $\alpha$ can be used to estimate the pivot energy (Zdziarski et al. 2003 and Figure 1 of Taylor et al. 2003).

Figure 3 (top) shows the hard $(2.0-10.0 \mathrm{keV})$ versus soft $(0.3-1.0 \mathrm{keV})$ count rates (flux-flux plot) for NGC 3783 from the $1 \mathrm{ks}$ binned XIS light curves. The scatter is partly due to poor statistics and is mostly removed when we bin the data. The red line is the best linear model which is summarized in Table 1 . Although visually the linear relationship appears correct, the fit is not statistically acceptable, with $\chi^{2} / \nu=40.4 / 12$. When we add a systematic error of $2 \%$ to each XIS count (as per Noda et al. 2011), the resulting fit gives $\chi^{2} / v=11.5 / 12$. 

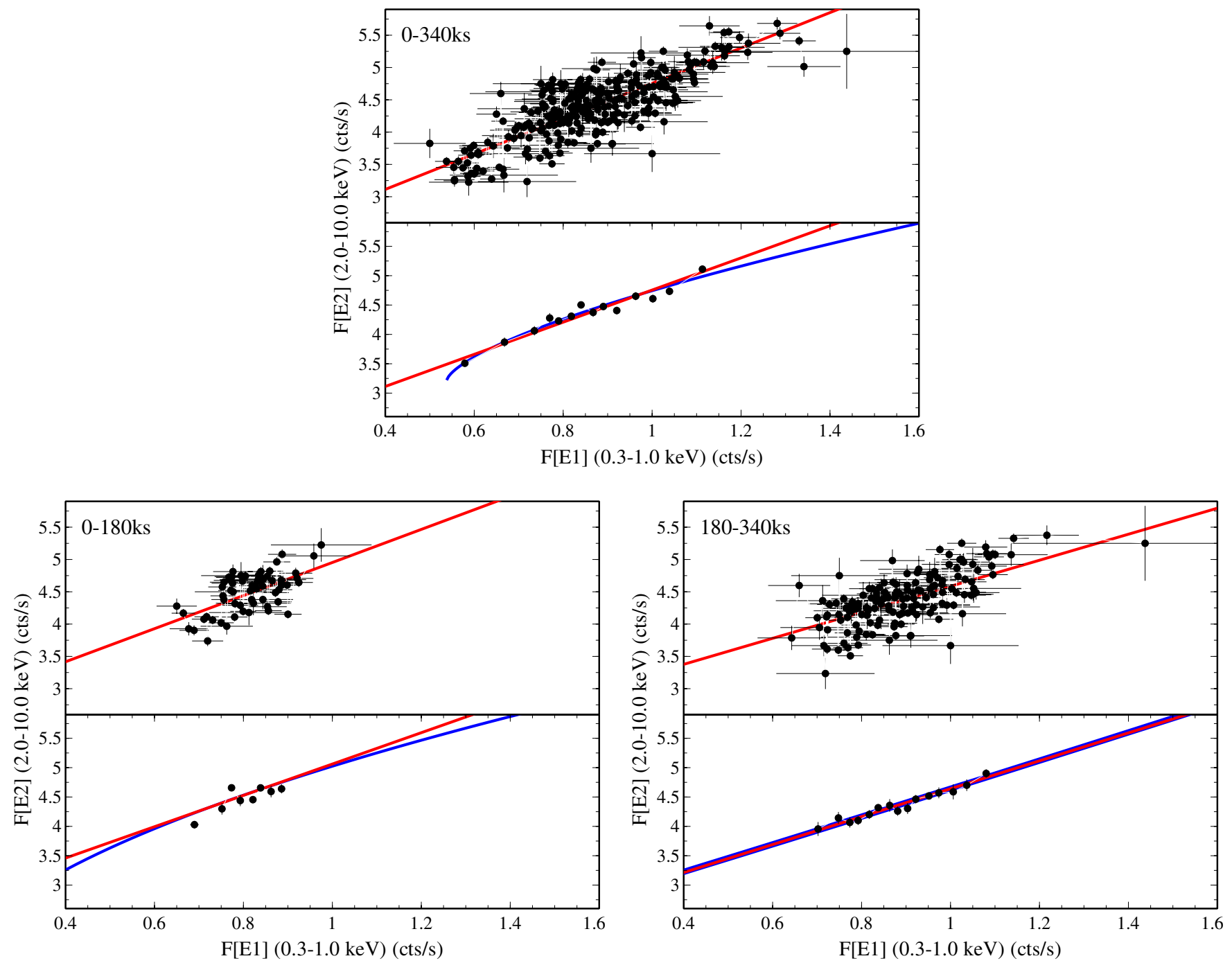

Figure 3. Flux-flux plots for NGC 3783 . Hard $(2.0-10.0 \mathrm{keV})$ vs. soft $(0.3-1.0 \mathrm{keV})$ count rate. Top panels: the points are from the light curve having $1 \mathrm{ks}$ time bins. Bottom panels: same as above but after averaging over the points and adding $2 \%$ systematic to each XIS count. In all cases, the red line shows the best linear fit to the data and the blue curve represents the best fit described by Equation (1).

(A color version of this figure is available in the online journal.)

Table 1

Summary of Linear and Power-law Fits to the Flux-Flux Plots Shown in Figure 3

\begin{tabular}{lccccccc}
\hline \hline Period & \multicolumn{3}{c}{ Linear Model } & & \multicolumn{3}{c}{ Power-law Model $^{\mathrm{a}}$} \\
\cline { 2 - 4 } \cline { 6 - 8 } & $k_{\text {lin }}$ & $C$ & $\chi^{2} /$ dof & & $k_{\text {pow }}$ & $\alpha$ & $\chi^{2} /$ dof \\
\hline Full range & $2.7 \pm 0.2$ & $2.1 \pm 0.2$ & $11.5 / 12$ & & $2.5 \pm 0.2$ & $0.63 \pm 0.16$ & $8.2 / 10$ \\
$0-180 \mathrm{ks}$ & $2.96 \pm 0.86$ & $2.1 \pm 0.7$ & $9.9 / 6$ & & $4.3 \pm 0.3$ & $0.55 \pm 0.22$ & $9.5 / 4$ \\
$180-340 \mathrm{ks}$ & $2.4 \pm 0.2$ & $2.3 \pm 0.1$ & $7.3 / 13$ & & $2.2 \pm 0.2$ & $1.05 \pm 0.07$ & $7.2 / 11$ \\
\hline
\end{tabular}

Notes. All errors are $1 \sigma$.

${ }^{a}$ The power law is of the type shown in Equation (1).

This extra systematic is likely due to uncertainties in CCD contamination (Noda et al. 2011). Replacing the linear relation with Equation (1) results in only a mild improvement $\left(\chi^{2} / v=\right.$ $8.2 / 10$; blue curve). This best fit cannot rule out weak pivoting nor does it statistically require it $(\alpha=0.63 \pm 0.16$ with constant offsets on the hard and soft axes of $C_{\mathrm{h}}=3.2 \pm 0.2$ and $C_{\mathrm{s}}=0.54 \pm 0.02$, respectively; $\left.1 \sigma\right)$. According to Figure 1 of Taylor et al. (2003), $\alpha$ ranging from approximately 0.55 to 0.8 implies a power law with a pivot energy ranging from 30 to $300 \mathrm{keV}$. In Section 3.4 we show using time-resolved spectra, evidence for a distinct change in the spectrum between the first and second half of the observation. In order to investigate whether any change is apparent in the flux-flux plot between these two time intervals, we created flux-flux plots for the first $180 \mathrm{ks}(\mathrm{P} 1+\mathrm{P} 2+\mathrm{P} 3$ in Figure 2) and the latter $160 \mathrm{ks}$ (P4+P5+P6). These are shown Figure 3 (bottom left and right, respectively) and the fits are summarized in Table 1.

The total flux range probed in this observation is quite modest $(\approx 1.8$ between highest and lowest flux bins) compared with other Seyfert 1s such as MCG-6-30-15 (factor of three; Vaughan 


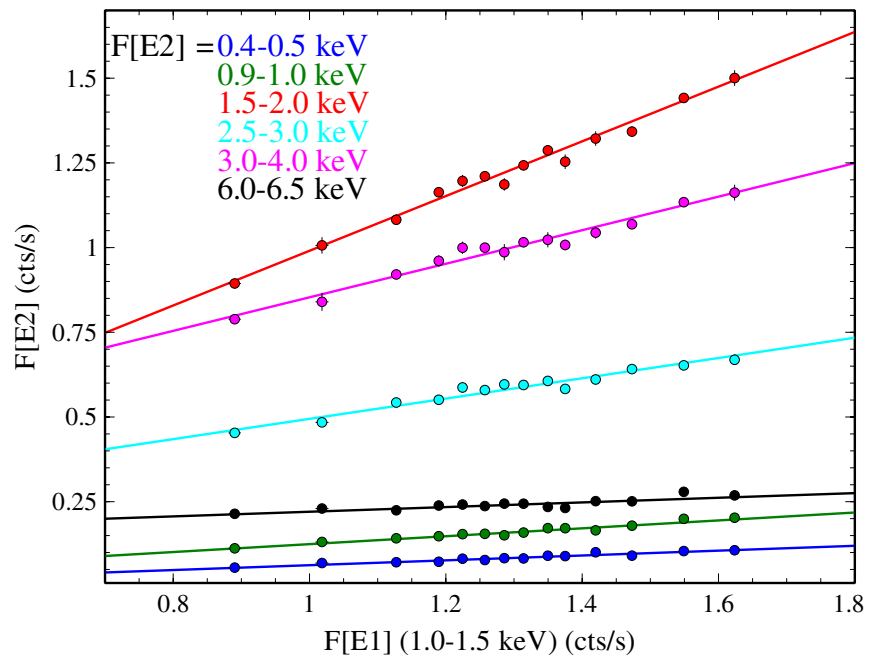

Figure 4. Binned band flux $F(\mathrm{E} 2)$ vs. $1.0-1.5 \mathrm{keV}$ flux. The various lines show the best linear fit to a sample of energy range. By obtaining the constant offset for the various energies with respect to a standard range, we can deduce the shape of the constant component (Section 3.2; see Figure 5).

(A color version of this figure is available in the online journal.)

\& Fabian 2004) or NGC 4051 (factor of eight over longer timescales; Uttley et al. 2004). This limited flux range prevents strong constraints on the derived value of $\alpha$, as is clear from the results shown in Table 1.

Under the assumption that the spectral variability in NGC 3783 can be described by the sum of a constant and variable component where the variable component has a constant shape, i.e., $\alpha=1$ in Equation (1), we can use flux-flux plots for a variety of energy bands with respect to a single reference band (Figure 4) to obtain the spectral shape of the constant component. Since the best fits are indeed roughly consistent with $\alpha=1$, this assumption is probably valid. Taylor et al. (2003) and Vaughan \& Fabian (2004) describe the methodology used here in detail.

On inspection, the reference band, $F(\mathrm{E} 1)$, is chosen to be the $1.0-1.5 \mathrm{keV}$ energy range as this shows the least contribution from the constant component (see Figure 5 and Figure 14). By measuring the offset of the flux-flux plot as a function of the discrete energy band $c(E)$, we can estimate the fractional contribution of the constant component to the spectrum as a function of energy. Figure 5 shows the offset $c$ as a function of energy. The offset was normalized relative to the average flux in each specific energy band. This normalization is applied so as to show the shape of the constant component independent of the instrument response. Note that by virtue of choosing the $1.0-1.5 \mathrm{keV}$ as the reference band, we are assuming that this band contributes little to the constant component, i.e., $c(1.0-1.5 \mathrm{keV}) \approx 0$. If, on the other hand, there is indeed a non-negligible contribution from the constant component to the overall spectrum at this energy range, then we should expect the scale to increase. However, the overall shape of the constant component will remain the same.

From Figure 5 it can be seen that the constant component is significantly harder than the total spectrum in the $2.0-10.0 \mathrm{keV}$ band. A similar analysis for MCG-6-30-15 (Vaughan \& Fabian 2004, see their Figure 11) shows that the constant component in MCG-6-30-15 has an increasingly larger amplitude as the energy softens below $1 \mathrm{keV}$. This is not the case for NGC 3783 where the constant component seems to flatten in this energy range. Furthermore, we note the presence of a feature at around

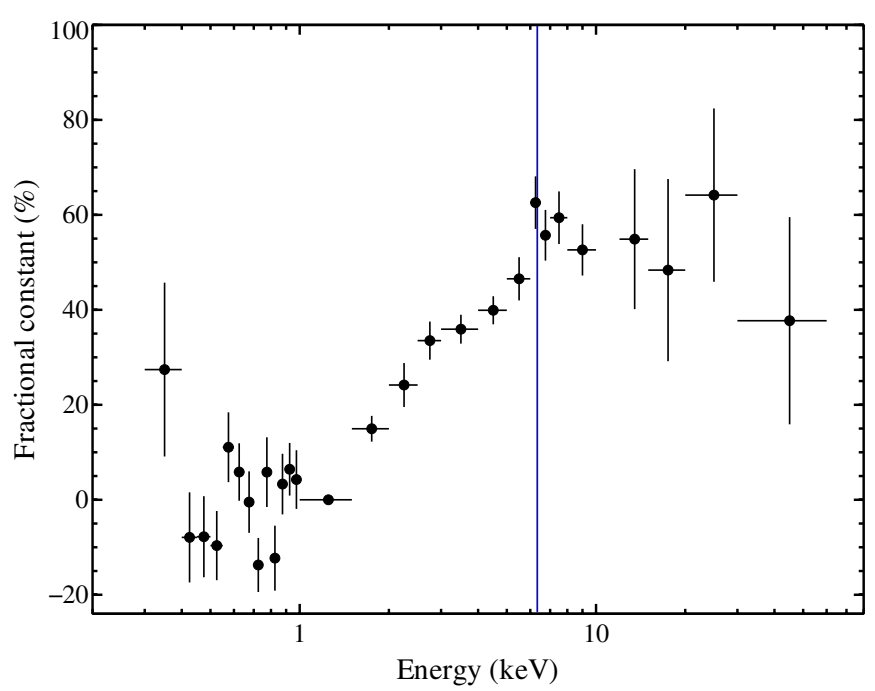

Figure 5. Shape of the constant component as obtained from the linear flux-flux relations shown in Figure 4. The constant vertical offsets were divided by the flux in each energy bin so as to show the shape of the constant component independent of the instrumental response. The vertical line shows the expected position of the iron $\mathrm{K} \alpha$ emission line at $6.4 \mathrm{keV}$ (rest frame).

(A color version of this figure is available in the online journal.)

$6 \mathrm{keV}$ (indicated by the solid vertical line in Figure 5) in the spectrum of the constant component as well as a further flattening of the spectrum above $10 \mathrm{keV}$. These features are qualitatively very similar to that found for MCG-6-30-15 by Taylor et al. (2003, see their Figure 5) where the authors identified the constant component as reflection from the inner accretion disk, similar to what was proposed by Fabian \& Vaughan (2003).

It should be stressed that the "constant component" identified via this model-independent method and shown in Figure 5 needs not be a single component nor does it need to be truly constant. It is plausible that this component be in fact the superposition of two distinct components whose variability are significantly weaker than that of the "variable" component.

As shown in Paper I, the time-averaged spectrum of NGC 3783 displays a fairly strong narrow $6.4 \mathrm{keV}$ emission line; this is a strong indication for X-ray reflection from cold and distant material. While the exact location of this material is unclear, the limits on the width of the line (FWHM $<5500 \mathrm{~km} \mathrm{~s}^{-1}$; Shu et al. 2010) under the assumption of dynamical equilibrium, place this cold material at a distance of $r>10^{4} r_{g}$ from the black hole. For a mass of $M=3 \times 10^{7} M_{\odot}$ (Vestergaard \& Peterson 2006 quote values ranging from $M=(2.8-3.0) \times$ $10^{7} M_{\odot}$ based on optical single-epoch spectroscopy), this places the cold reflector at $r>4.4 \times 10^{16} \mathrm{~cm}$ which corresponds to 17 lt-day or more. Thus, during the span of this observation, it is expected that the distant/cold reflection component will be approximately constant. Some, but not all, of the constant component revealed in Figure 5 can be identified with this distant reflection.

\subsection{Flux-resolved Spectroscopy}

A further way to explore the X-ray variability is by examining the evolution of the energy spectra with flux. To do this we divided the data into five flux intervals (F1-F5 shown in Figure 6) each having approximately the same number of photons $\left(\sim 2.2 \times 10^{5}\right.$ and $1.4 \times 10^{5}$ for the BI and FI, respectively). Spectra and responses were produced for each flux 


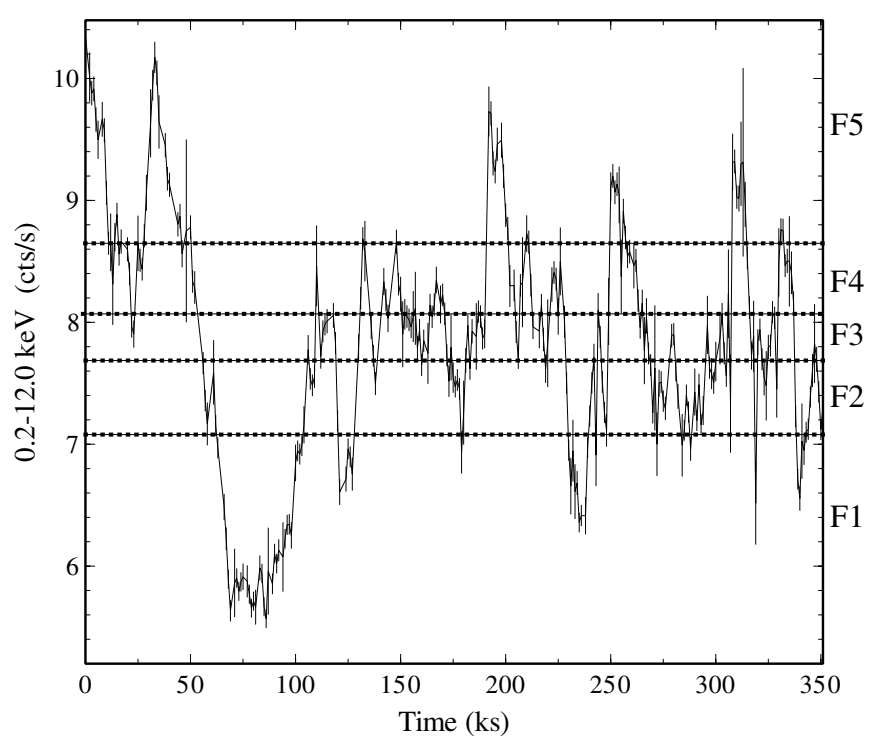

Figure 6. $0.3-12 \mathrm{keV}$ light curve (1 ks bin) showing the various flux bins used in the following section.

interval. To illustrate the spectral change as a function of flux we show in Figure 7 both the high (F5; black) and low (F1; red) spectra as ratio to an absorbed $\left(N_{\mathrm{H}}=9.91 \times 10^{20} \mathrm{~cm}^{-2}\right)$ power law having $\Gamma=1.8$. It is clear that at low flux the spectrum is harder above $2 \mathrm{keV}$, which is as expected from the twocomponent model described in the previous section. Assuming the spectrum is composed of a soft-variable component together with a hard-constant component (similar to that shown in Figure 5) then as the total flux decreases, the relative contribution of the harder component increases. This behavior is also seen in other Seyfert galaxies such as MCG-6-30-15 (Vaughan \& Fabian 2004, and references therein).

\subsubsection{Difference-spectra}

In this section we use the spectra obtained from the five flux levels described above to produce flux-resolved "differencespectra." By subtracting the lowest flux spectrum (F1) from the remaining spectra, we effectively remove the contribution of any constant (emission) component. This technique was described in detail in Fabian \& Vaughan (2003) and results in a spectrum consisting of the variable component modified by any intervening (Galactic and intrinsic) absorption.

In subtracting the XIS spectra we assume that the background for each period is constant and therefore use the differencespectrum without background subtraction. To test this assumption we investigated the background in the XIS-FI data over the five periods described in Section 3.4. In each period, the $0.5-10$ background count rate is found to be approximately $3 \times 10^{-2}$ count s $^{-1}$, which, in all cases, corresponds to less that $1.5 \%$ of the total source rate. Similarly, the $8-10 \mathrm{keV}$ background count rate, although slightly more variable (ranging from $2.3 \times 10^{-3}$ count s $^{-1}$ to $2.7 \times 10^{-3}$ count $\mathrm{s}^{-1}$ ), always remains at less than $5 \%$ of the total source counts. However, due to the strong time dependency of the PIN background, we produced background-subtracted spectra for each period and then created the difference-spectra using MATHPHA, propagating the errors in each arithmetic operation. Figure 8 (left) shows the data/model ratio for the four independent fluxresolved difference-spectra fitted with an absorbed power law above $3 \mathrm{keV}$. Such an absorbed power law results in a satis-

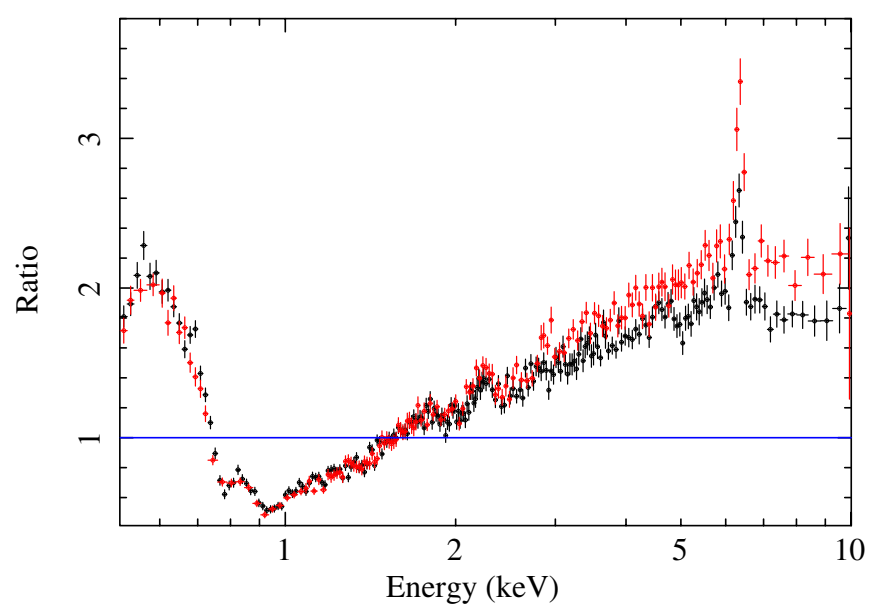

Figure 7. Ratio of high (F5; black) and low (F1; red) flux BI spectrum to an absorbed $(\Gamma=1.8)$ power law. The shape of the soft energy range is remarkably similar in both cases suggesting that the warm absorber is not changing. As is usually seen in other sources (e.g., MCG-6-30-15), the overall spectrum becomes harder with a more prominent iron feature at low fluxes. This is as expected from the two-component model described in Section 3.2.

(A color version of this figure is available in the online journal.)

factory fit with $\chi^{2} / v=1716.5 / 1669(1.03)$ above $3 \mathrm{keV}$, with $\Gamma=1.86 \pm 0.05$ at the $90 \%$ level of confidence. Allowing $\Gamma$ to vary between the various difference-spectra did not result in a statistically significant improvement to the overall fit, with a decrease in $\chi^{2}$ of 7.8 for three degrees of freedom (dof; $F$-test value of 2.5). Table 2 details the values found for $\Gamma$ for the four difference-spectra as well as the global value shown above. When we extend the data to cover the full range, we see clear flux deficit below $2 \mathrm{keV}$ due to the warm absorber, which is not removed in the difference-spectra.

In order to model the warm absorber we use the same photoionization model as in Paper I (generated using XSTAR v2.2.0). The free parameters of this absorption model are the column density and ionization parameter of the absorbing medium. Following from the results of the time-averaged spectrum presented in Paper I, we have modeled the warm absorber assuming three distinct zones. To reduce computational time we have frozen the ionization parameter of the various zones to the values found in Paper I. We have also investigated varying the ionization parameters while fixing the column densities to the values reported in Paper I, however it was found to give similar results and so we chose to carry on with the former approach. Because the Suzaku/XIS detectors do not have the spectral resolution capable of constraining the outflow velocities of the various warm absorbers zones, we follow the practice employed in Paper I and hold the redshifts of these components fixed at the cosmological value for NGC 3783. As such, in each period the free parameters of the model were the normalization of the power law together with the column densities for the three absorbing zones. Figure 8 (right) shows the $\mathrm{data} /$ model ratio for the various difference-spectra after the inclusion of the three-zone warm absorber. The best fit resulted in $\chi^{2} / v=2995.3 / 2853(1.05)$. Figure 9 shows the fit to the difference-spectra resulting from the subtraction of the bottom two flux bins from the top two flux bins (see Figure 6). A simple power law $(\Gamma=1.94 \pm 0.05)$ modified by a three-zone warm absorber again results in an excellent fit to the difference-spectra $\left(\chi^{2} / v=753.6 / 709(1.06)\right)$. This fit to the full energy spectrum is also summarized in Table 2 . The analysis presented in this section re-enforces the result of the flux-flux analysis (Section 3.2) 

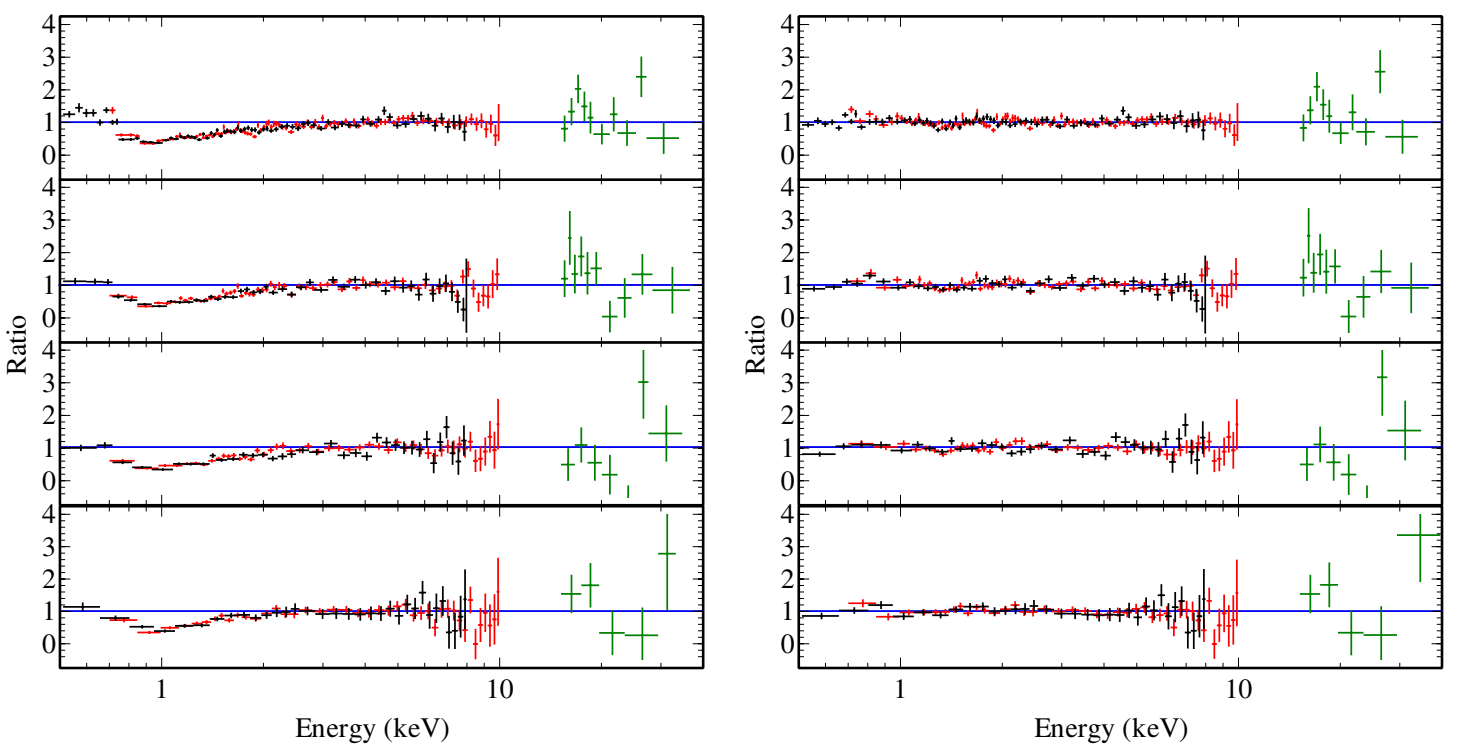

Figure 8. Left: data/model ratio of the flux-resolved difference-spectra to an absorbed power law. The difference-spectra shown are (from top to bottom) F5, F4, F3, and F2 minus F1. The spectra were fit above $3 \mathrm{keV}$ with a power law and extended to the full energy range after the inclusion of neutral Galactic absorption. BI, FI, and PIN data are shown in black, red, and green, respectively. Right: now with the inclusion of a three-zone warm absorber.

(A color version of this figure is available in the online journal.)

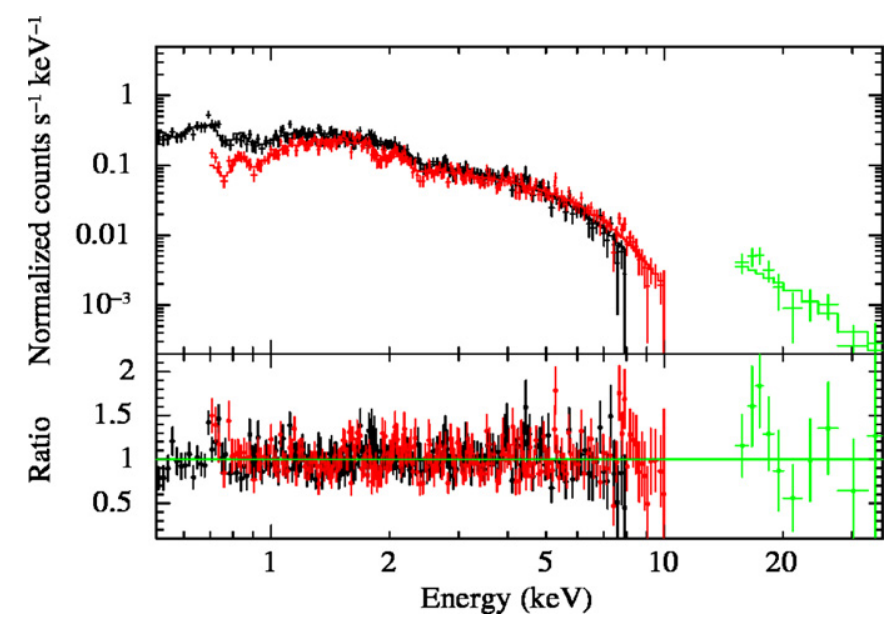

Figure 9. Similar model to Figure 8 (right) fitted to the difference-spectra resulting from the subtraction of the bottom two flux bins from the top two flux bins (see Figure 6).

(A color version of this figure is available in the online journal.) that the shape of the variable component (the power law in this case) does not vary significantly with flux.

\subsection{Time-resolved Spectroscopy}

In order to further visualize the variability we divided the full observation into seventeen $20 \mathrm{ks}$ segments (real time) and extracted spectra and responses for each time segment. Each spectrum was then compared to the time-averaged spectrum by taking the ratio between them. The result was the ratio-at all energies-between the time-averaged spectrum and each $20 \mathrm{ks}$ segment. Figure 10 shows this ratio as a function of time and energy (top), together with the total count rate for each segment (bottom). Apparent changes to the overall spectrum are seen at approximately 60,180 , and $260 \mathrm{ks}$, with some indications of a further discontinuity at $300 \mathrm{ks}$. We note here that from Figure 10 it appears possible that there are narrow regions showing spectral variability at timescales shorter than $20 \mathrm{ks}$ (e.g., halfway through the fourth period the color code between $5-7 \mathrm{keV}$ changed from orange to green implying a significance greater than $3 \sigma$ ), however spectra extracted at these timescales have very low

Table 2

Summary of Fits to the Various Flux-resolved iDifference-spectra

\begin{tabular}{lcccc}
\hline \hline \multicolumn{5}{c}{ Flux-resolved Difference-spectra } \\
\hline Flux Bin $^{\mathrm{a}}$ & \multicolumn{4}{c}{ Full Range $^{\mathrm{c}}$} \\
\hline & Gamma Untied & Gamma Tied & Gamma Tied & Gamma Tied \\
F5-F1 & $1.8 \pm 0.1$ & $1.86 \pm 0.05$ & $2.00 \pm 0.06$ & $\ldots$ \\
F4-F1 & $1.95 \pm 0.1$ & $\ldots$ & $\ldots$ & $\ldots$ \\
F3-F1 & $1.92 \pm 0.13$ & $\ldots$ & $\ldots$ & $\ldots$ \\
F2-F1 & $2.0 \pm 0.2$ & $\ldots$ & $\ldots$ & $\ldots$ \\
T-B & $\ldots$ & $\ldots$ & $\ldots$ & $1.94 \pm 0.05$ \\
$\chi^{2} / v$ & $1708.7 / 1666(1.03)$ & $1716.5 / 1669(1.03)$ & $2995.3 / 2853(1.05)$ & $753.6 / 709(1.06)$ \\
\hline
\end{tabular}

Notes.

${ }^{a}$ The flux bins refer to the various flux slices shown in Figure 6.

$\mathrm{b}$ The data were fit with a simple absorbed power law in the $3-40 \mathrm{keV}$ energy range.

${ }^{c}$ The full $0.5-40 \mathrm{keV}$ energy range was fit after the inclusion of a three-zone warm absorber.

${ }^{\mathrm{d}} \mathrm{T}$ and $\mathrm{B}$ refers to flux bins F5 + F4 and F1 + F2, respectively. All errors refer to the $90 \%$ confidence range. 

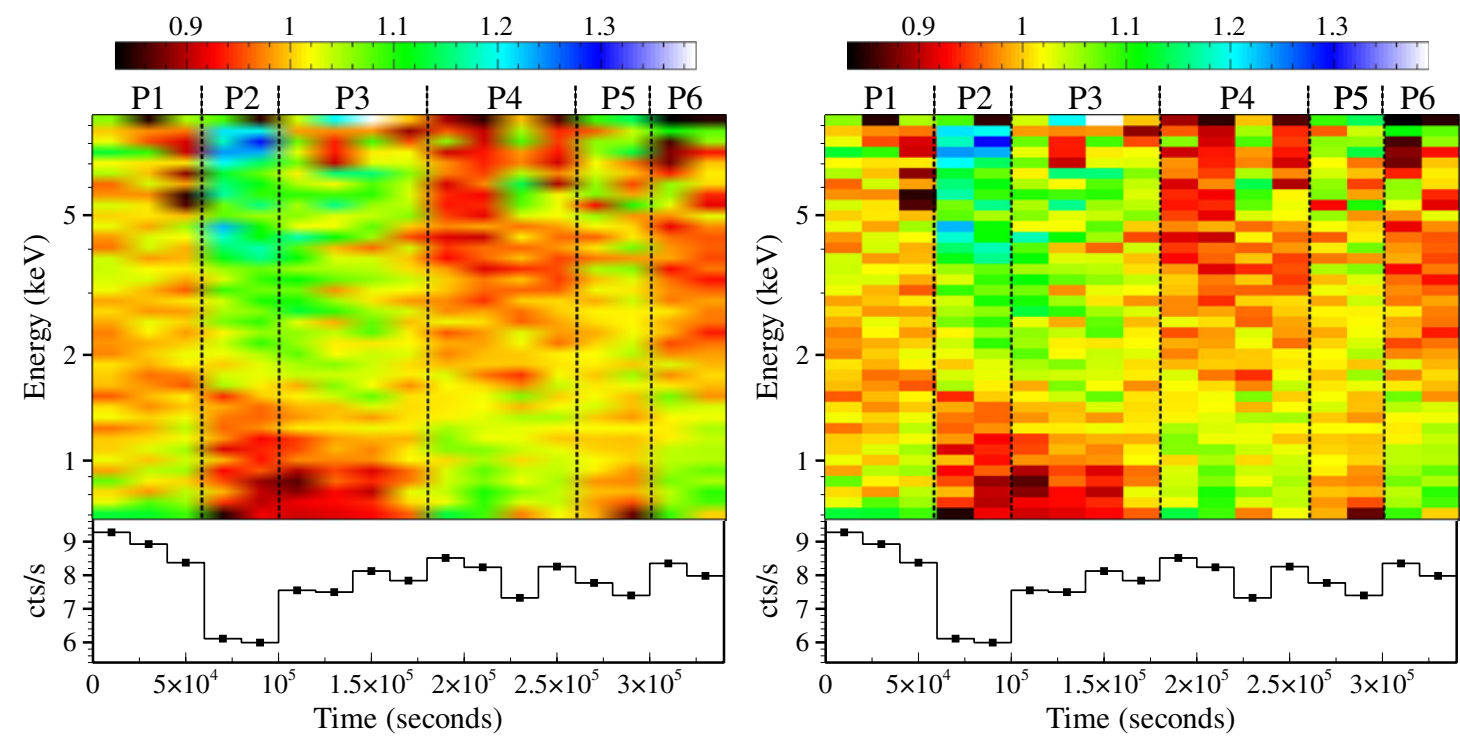

Figure 10. Smoothed (left) and un-smoothed (right) time-resolved spectra for $20 \mathrm{ks}$ segments. Top: two-dimensional image showing the ratio of the various spectra to the time-averaged spectrum as a function of energy and time. The typical standard deviation is approximately 0.03 . Bottom: corresponding XIS count rate. The vertical dotted lines show the position of possible changes in the spectral shape as compared to the average. Based on this image and Figure 2, we divide the total observation into six periods.

(A color version of this figure is available in the online journal.)
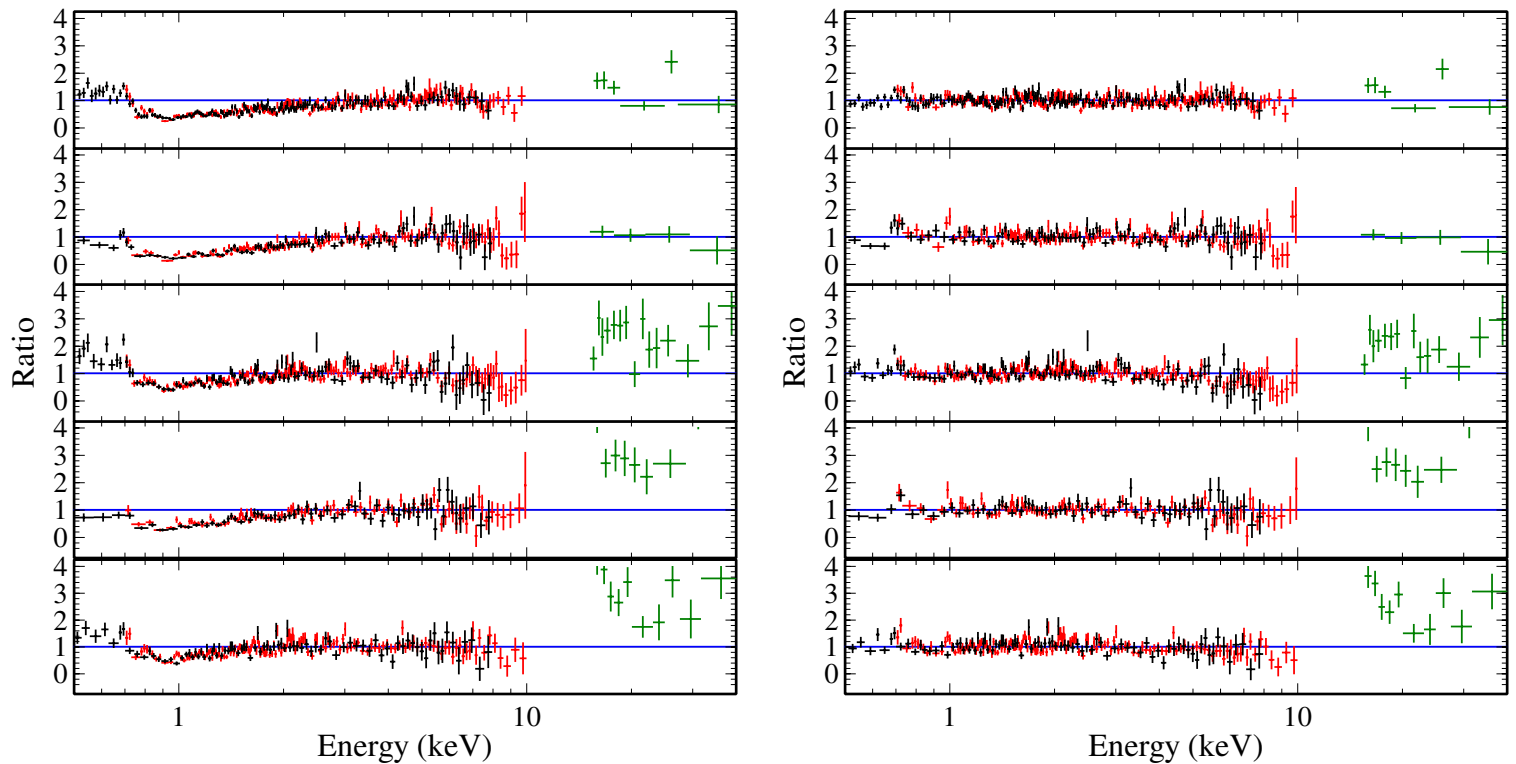

Figure 11. Left: data/model ratio for the difference-spectra between (from top to bottom) P1, P3, P4, P5, and P6 minus P2. The difference-spectra shown on the left are fit between 3 and $10 \mathrm{keV}$ with a power law and extended to the full energy range. Right: after the inclusion of a three-zone warm absorber fitted between 0.5-10 keV. In both cases, the P4, P5, and P6 difference-spectra show a clear hard excess that is not an artifact of modeling the ionized absorber. BI, FI, and PIN data are shown in black, red, and green, respectively.

(A color version of this figure is available in the online journal.)

signal to noise, which prevents us from performing meaningful spectral decomposition. We stress that this image is employed only to show the general trend in the energy-time parameter space. Based on the broad trends apparent in Figure 10 and the light curve shown in Figure 2, we break the full observation into six distinct spectral periods (P1-P6) in order to carry out a similar analysis to that of Section 3.3 and produce differencespectra for the five time periods with respect to the period with the lowest count rate (P2 in Figure 2).

\subsubsection{Difference Spectra}

Figure 11 (left) shows the data/model ratio for the five timeresolved difference-spectra fitted with an absorbed power law above $3 \mathrm{keV}$. For the top two difference-spectra (P1 and P3 minus P2), a power law with an index fixed 1.95, consistent with the value found in the previous section, was satisfactory, with $\chi^{2} / v=686.0 / 726(0.94)$. Allowing $\Gamma$ to vary resulted in slight improvement to the fit $\left(\Delta \chi^{2}=15.6\right)$ and a lower value for the power-law index, with $\Gamma=1.80 \pm 0.06$. When we add the latter three difference-spectra (P4, P5, and P6 minus P2), we find that, whereas the $3-10 \mathrm{keV}$ range is well fit with a power law having an index of $\sim 1.95$, the flux above $10 \mathrm{keV}$ is severely underpredicted. This is highlighted in the bottom two panels of Figure 11 (left) where $\Gamma=1.95$. Allowing $\Gamma$ to vary in the second half of the observation resulted in a statistically acceptable fit $\left(\chi^{2} / v=1793.7 / 1747(1.03)\right)$, however it required 
Table 3

Summary of Fits to the Various Time-resolved Difference-spectra

\begin{tabular}{lcc}
\hline \hline & \multicolumn{2}{c}{ Time-resolved Difference-spectra $^{\mathrm{a}}$} \\
\hline Period $^{\mathrm{b}}$ & Gamma Untied & Gamma Tied \\
$\mathrm{P} 1-\mathrm{P} 2$ & $1.95 \pm 0.02$ & $1.95 \pm 0.05$ \\
$\mathrm{P} 3-\mathrm{P} 2$ & $1.95_{-0.03}^{+0.02}$ & $\ldots$ \\
$\mathrm{P} 4-\mathrm{P} 2$ & $2.01_{-0.02}^{+0.04}$ & $\ldots$ \\
$\mathrm{P} 5-\mathrm{P} 2$ & $1.68_{-0.03}^{+0.05}$ & $\ldots$ \\
$\mathrm{P} 6-\mathrm{P} 2$ & $1.87_{-0.02}^{+0.06}$ & $\ldots$ \\
$\chi^{2} / v$ & $3496.7 / 3224$ & $3518.5 / 3228$ \\
\hline
\end{tabular}

Notes.

a All cases refer to fits performed in the full $0.5-40 \mathrm{keV}$ energy range.

b The difference-spectra are made from the periods highlighted in Figures 2 and 10. This model successfully accounts for the PIN data during the first half of the observation but severely underpredicts the high-energy flux in the difference-spectra in the latter half.

uncharacteristically low values for $\Gamma$ of $\approx 1.38-1.44$. Indeed, if we replace the single power law with a broken-power model having a global gamma below $10 \mathrm{keV}\left(\Gamma_{<10 \mathrm{keV}}\right)$ and allow $\Gamma_{>10 \mathrm{keV}}$ to vary between the periods, the various differencespectra, including the hard excesses, can be phenomenologically modeled $\left(\chi^{2} / v=1687.5 / 1744\right)$ with $\Gamma_{<10 \mathrm{kev}}=1.88_{-0.07}^{+0.06}$ and $\Gamma_{>10 \mathrm{keV}}=1.8 \pm 0.2,1.9 \pm 0.3,1.07_{-0.15}^{+0.17}, 0.73_{-0.13}^{+0.16}$, and $0.87_{-0.13}^{+0.16}$ for P1, P3, P4, P5, and P6 minus P2, respectively. It is clear that, whereas the first half of the observation does not require a break in the power law, the second half requires the presence of an extra hard component.

Figure 11 (right) shows the data/model ratio for the various difference-spectra after the inclusion of a three-zone warm absorber similar to that of the previous section (Section 3.3). The fit was obtained by modeling simultaneously all spectra in the XIS energy range $(0.5-10 \mathrm{keV})$ together with the PIN data for periods 1 and 3 only. The PIN data for periods 4, 5, and 6 emphasize the level of excess above the unbroken powerlaw continuum. The best-fit model consisting of a $(\Gamma=1.95)$ power-law fit to the full energy range but excluding the PIN data for the second half of the observation, resulted in $\chi^{2} / v=$ $3198.7 / 3189$ (1.00). Including the PIN data for periods 4,5 , and 6 results in a worst fit $\left(\chi^{2} / v=3518.5 / 3228(1.09)\right)$ with the excess above $10 \mathrm{keV}$ being the prominent source of residuals. Allowing the power-law indices to vary between the various difference-spectra did not remove the hard excess in the latter half of the observation. We show in Table 3 the values obtained for $\Gamma$ as a function of time using this simple absorbed powerlaw model. We emphasize that since there is still a distinct hard excess in the latter half of the observation, these values are only illustrative.

The presence of such an excess at hard energies $(>10.0 \mathrm{keV})$ in the difference-spectrum implies that there must be a further varying component whose contribution is greatest at these energies. Furthermore, this variation does not occur monotonically with flux, since such hard excesses were not present in the various difference-spectra presented in Section 3.3. The fact that in all difference-spectra the energy range below $\approx 10 \mathrm{keV}$ was well modeled with a simple power law further suggests that this extra component contributes little to the variability below this energy. A possible origin for the spectral variation at high energies as a function of time could be due to change in the ionization state of the innermost accretion disk and/or change in the contribution to the spectrum from the reflection component. The latter can be parameterized by the ratio $\mathcal{R}$ between the reflection and illuminating power-law flux. This possibility will be investigated further in the following section.

\subsection{Fitting the Individual Time Segments}

We have shown in the previous sections that the differencespectra-a rough indication of the variable components in the total observed spectrum - can be successfully modeled using a simple power law above $3 \mathrm{keV}$ during all flux levels; however, when the spectrum is broken down in time, this simple power law does not describe the latter half of the observation. The difference-spectrum for the second half of the observation requires a further variable component that imprints itself most notably at hard energies. In all cases, when the power-law continuum is extended to lower energies it reveals the strong presence of a warm absorber whose ionization and column density does not vary with time or flux. A self-consistent origin for both the constant hard component as well as the varying component above $10 \mathrm{keV}$ (other than the varying power law) lies in the intrinsic shape of a reflection continuum arising from the innermost regions around a black hole for a variety of ionizations. In the following we adopt such a reflection component as the origin of both the hard-constant component and the further variability over the power-law component above $10 \mathrm{keV}$. We note that a reflection continuum as such is indeed usually invoked to explain the hard component in various Seyfert 1s AGNs (see, e.g., Zoghbi et al. 2010; Walton et al. 2010).

\subsubsection{Three-component Model}

In order to model the individual time segments as shown in Figure 2, we construct a model based on the primary source of the intrinsic power-law continuum being located in a region of strong gravity, and gravitational light bending focusing some of the emission from this component down onto the disk. The reflection component was calculated with the REFLIONX code by Ross \& Fabian (2005), convolved with the KDBLUR kernel (Laor 1991) to account for the relativistic effects present close to the black hole. In Paper I, we used the highly sophisticated variable-spin relativistic smearing model RELCONV (Dauser et al. 2010) as the goal was to constrain the spin parameter. However, for this study of broadband X-ray variability, such a complex convolution model results in very large computational time with no extra benefit beyond that which KDBLUR can provide.

We assume a radial dependence for the emissivity index and ionization parameter of the disk, such that within $r_{\mathrm{br}}$ the disk is highly ionized with a variable ionization parameter resulting in reflection which is modeled with an inner, hot, relativistically convolved REFLIONX, and beyond this radius it has a constant value of $\xi=1 \mathrm{erg} \mathrm{cm} \mathrm{s}^{-1}$ up to an outer radius fixed at $400 r_{\mathrm{g}}$ (modeled with an outer, cold, blurred REFLIONX). After several trials, we fixed the break radius at $30 r_{\mathrm{g}}$. Changing this value to anywhere greater than approximately $6 r_{\mathrm{g}}$ does not change the results presented below. To account for the narrow Fe XXVI emission line present in the spectra, we add a Gaussian centered at $6.97 \mathrm{keV}$ with $\sigma=10 \mathrm{eV}$. The normalization of this line is kept tied between each period and we find its equivalent width to vary between approximately $27 \mathrm{eV}$ and $36 \mathrm{eV}$, in close agreement to the value found in the time average spectrum $\left(W_{\text {Fe xxvI }}=22 \pm 5 \mathrm{eV}\right.$; Paper I). Reflection from distant, low-velocity, cold matter is modeled with PEXRAV (Magdziarz \& Zdziarski 1995) together with a further narrow $\mathrm{Fe} \mathrm{K} \alpha$ Gaussian at $6.4 \mathrm{keV}$. When employing this model, the 
reflection fraction and folding energy of the cold reflector were frozen at $\mathcal{R}_{\text {cold }}=0.5$ and $200 \mathrm{keV}$, respectively, similar to the values found in Paper I. We note here that this model differs slightly from that employed in Paper I in that the previous paper used a superior model (PEXMON; Nandra et al. 2007) which self-consistently accounts for the Compton backscattered reflection continuum as well as the $\mathrm{K} \alpha$ and $\mathrm{K} \beta$ emission lines of iron, the Compton shoulder of the iron $\mathrm{K} \alpha$ line, and the $\mathrm{K} \alpha$ line of nickel. These fine details are extremely important when modeling the narrow energy range around the broad iron line and thus determining spin, however for the broadband variability we are interested in this work, the commonly used combination of PEXRAV+GAUSSIAN suffices. In all fits, the equivalent width of the iron $\mathrm{K} \alpha$ line lies in the $80-100 \mathrm{eV}$ range (compared with $W_{\mathrm{K} \alpha}=98 \pm 5 \mathrm{eV}$ and $88 \pm 6 \mathrm{eV}$ for Suzaku and Chandra data, respectively; Paper I).

Paper I investigated the effect of (1) fixing the iron abundance of the distant reflector (PEXRAV in our case) to that of the inner accretion disk, and (2) allowing these abundances to vary independently. The authors find that a high iron abundance $(\mathrm{Fe} /$ solar $\approx 4)$ is statistically preferred, however little difference is found between the two scenarios. Here we fix the abundance of the disk reflection (both inner and outer REFLIONX) at four times solar, similar to the value used in Paper I but keep the distant, cold reflector fixed at solar. The Galactic neutral hydrogen column density and inner disk inclination were fixed at $9.91 \times 10^{20} \mathrm{~cm}^{-2}$ and $23^{\circ}$ as found in Paper I. The power-law indices of both REFLIONX components are tied to that of the main continuum. The emissivity profile beyond $r_{\mathrm{br}}$ is assumed to follow $\epsilon(r) \propto r^{-3}$. We account for the effects of the threezone warm absorber by the use of the XSTAR grids described in Section 3.3 with the values for the ionization parameters frozen to that found in Paper I. Similarly to Paper I, we also allow a fraction $f$ of the continuum to scatter/leak around the warm absorber. This value has consistently been found to be around $15 \%$. Emission from a blackbody with temperature of $\approx 55 \mathrm{eV}$ is also included in the fit. The total model thus consists of seven global parameters - the inner disk radius together with the inner disk emissivity index, the power-law index $\Gamma$, blackbody normalization, normalizations of the two narrow emission lines

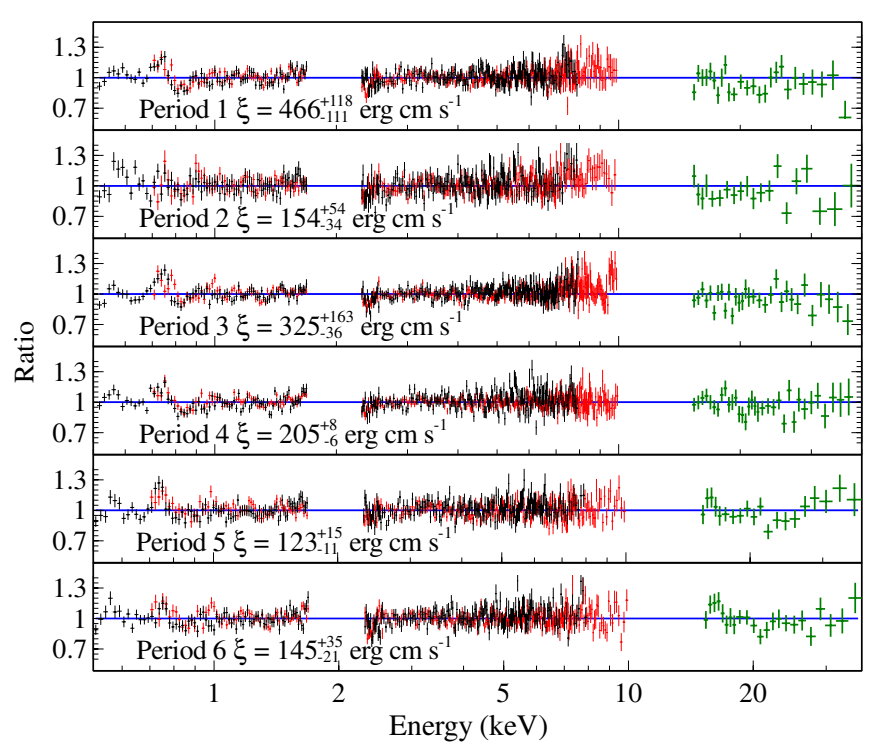

Figure 12. Data/model ratio for the six periods fitted simultaneously with a model consisting of a power law, a cold $\left(\xi=1 \mathrm{erg} \mathrm{cm} \mathrm{s}^{-1}\right)$ reflection component originating in the outer parts of the accretion disk, and a "hot" reflection component with variable ionization ( $\xi$ between $\sim 100-500 \mathrm{erg} \mathrm{cm} \mathrm{s}^{-1}$ shown in the figure) originating from the innermost regions of the accretion disk. The continuum is absorbed by the three-zone warm absorber described previously, and the fit includes two narrow emission lines (Fe $\mathrm{K} \alpha$ and $\mathrm{Fe} \mathrm{XXVI)} \mathrm{as} \mathrm{well} \mathrm{as}$ reflection from cold, low-velocity matter as described fully in Section 3.5.1. See Figure 14 for a visual depiction of the evolution of these three components during the different periods.

(A color version of this figure is available in the online journal.)

(Fe $\mathrm{K} \alpha$ and $\mathrm{Fe} \mathrm{XXVI}$ ), and the fraction of scattered continuum $f$. In addition to these seven global parameters, we also have a further seven parameters individual to each period-column densities of the three XSTAR grids, inner reflection ionization parameter, power-law normalization, and the normalization of both inner and outer reflection components. This model resulted in a good fit to all the individual periods $\left(\chi^{2} / v=4494.7 / 3372\right)$.

Figure 12 shows the data/model ratio for all six periods and Table 4 summarizes the values found for the various parameters

Table 4

Summary of Fit to the Various Time-resolved Spectra

\begin{tabular}{|c|c|c|c|c|c|c|}
\hline Parameter & $\mathrm{P} 1$ & $\mathrm{P} 2$ & P3 & $\mathrm{P} 4$ & P5 & P6 \\
\hline$N_{\mathrm{H}}\left(\mathrm{cm}^{-2}\right)^{\mathrm{a}}$ & $9.91 \times 10^{20}(\mathrm{f})$ & $\ldots$ & $\ldots$ & $\ldots$ & $\ldots$ & $\ldots$ \\
\hline$K T_{\mathrm{BB}}(\mathrm{eV})$ & 54.6(f) & $\ldots$ & $\ldots$ & $\ldots$ & $\ldots$ & $\ldots$ \\
\hline Flux $_{\mathrm{BB}}{ }^{\mathrm{b}}$ & $3.1 \pm 1.8$ & $\ldots$ & $\ldots$ & $\ldots$ & $\ldots$ & $\ldots$ \\
\hline$\Gamma$ & $1.82 \pm 0.01$ & $\ldots$ & $\ldots$ & $\ldots$ & $\ldots$ & $\ldots$ \\
\hline$q_{r} \leqslant r_{\mathrm{br}}$ & $4.3 \pm 0.4$ & $\ldots$ & $\ldots$ & $\ldots$ & $\ldots$ & $\ldots$ \\
\hline$r_{\text {in }}\left(r_{\mathrm{g}}\right)$ & $<1.4$ & $\ldots$ & $\ldots$ & $\ldots$ & $\ldots$ & $\ldots$ \\
\hline Inclination & $23(f)$ & $\ldots$ & $\ldots$ & $\ldots$ & $\ldots$ & $\ldots$ \\
\hline $\mathrm{Fe}\left(\mathrm{Z}_{\mathrm{Fe}}\right)$ & $4(f)$ & $\ldots$ & $\ldots$ & $\ldots$ & $\ldots$ & $\ldots$ \\
\hline$\xi\left(\mathrm{erg} \mathrm{cm} \mathrm{s}^{-1}\right)$ & $466_{-186}^{+44}$ & $148_{-33}^{+55}$ & $284_{-33}^{+192}$ & $205_{-13}^{+8}$ & $123_{-10}^{+15}$ & $145_{-16}^{+33}$ \\
\hline Flux $_{\text {power law }}$ & $37.2 \pm 0.7$ & $29.0 \pm 0.6$ & $34.2 \pm 0.6$ & $31.5_{-0.8}^{+0.7}$ & $30.9_{-0.6}^{+0.5}$ & $30.7_{-0.6}^{+0.5}$ \\
\hline Flux Inner ref. & $7.5_{-0.4}^{+0.6}$ & $2.4 \pm 0.6$ & $5.9_{0.3}^{+0.5}$ & $8.7 \pm 0.5$ & $8.9 \pm 0.9$ & $8.8 \pm 0.9$ \\
\hline Flux Outer ref. & $1.9 \pm 0.5$ & $2.8_{-0.7}^{+0.6}$ & $1.7 \pm 0.5$ & $1.0_{-0.4}^{+0.5}$ & $1.3 \pm 0.6$ & $1.6 \pm 0.6$ \\
\hline$\chi^{2} / v$ & $4497.7 / 3390(1.33)$ & $\ldots$ & $\ldots$ & $\ldots .4$ & $\ldots$ & $\ldots$ \\
\hline
\end{tabular}

Notes. Fit to the individual periods highlighted in Figures 2 and 10. The six periods were fit simultaneously with a number of global, as well as period-dependent, parameters. The model and the various parameters are described in Section 3.5.1.

a The hydrogen column density, disk temperature, inclination, and iron abundance are frozen (f) at the values quoted in Paper I.

$\mathrm{b}$ The various fluxes are found between 0.001 and $1000 \mathrm{keV}$ and are in units of $\times 10^{-11} \mathrm{erg} \mathrm{cm}^{-2} \mathrm{~s}^{-1}$. 

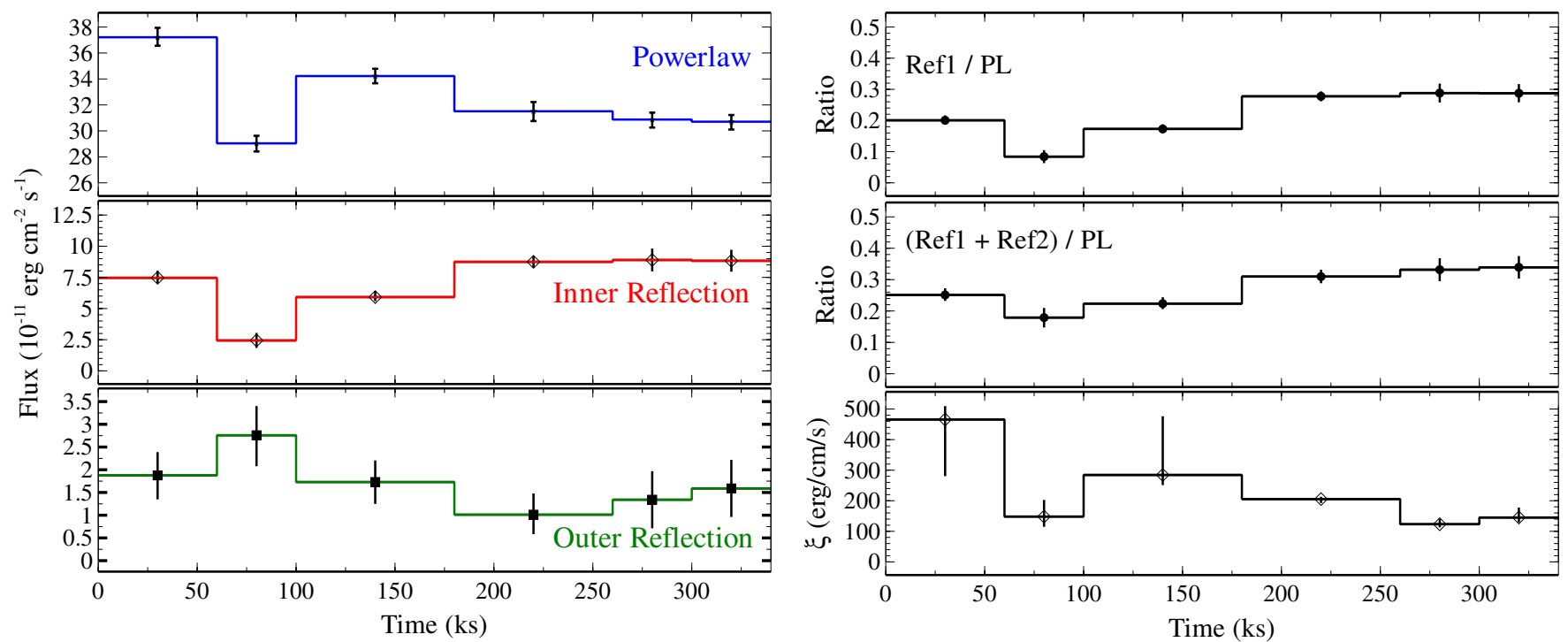

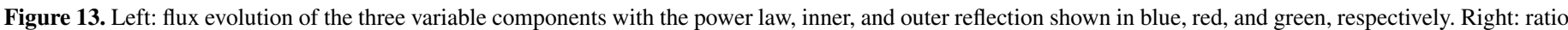
of the inner reflection (top) and total reflection (middle) to the power-law flux. The bottom panel shows the evolution of the ionization parameter $\xi$.

(A color version of this figure is available in the online journal.)

Table 5

Summary of Fits Having the Power Law Both Tied and Untied between the Six Periods

\begin{tabular}{|c|c|c|c|c|c|c|c|}
\hline Gamma & Period 1 & Period 2 & Period 3 & Period 4 & Period 5 & Period 6 & $\chi^{2} /$ dof \\
\hline Tied & \multicolumn{6}{|c|}{$1.82 \pm 0.01$} & $4497.7 / 3390$ \\
\hline Untied & $1.82 \pm 0.01$ & $1.82_{-0.03}^{+0.02}$ & $1.83_{-0.01}^{+0.02}$ & $1.83_{-0.01}^{+0.02}$ & $1.82_{-0.01}^{+0.02}$ & $1.83 \pm 0.02$ & $4487.4 / 3385$ \\
\hline
\end{tabular}

of interest. In order to obtain the errors (90\% confidence) for the various parameters of interest in this study-mainly the inner disk ionization, fluxes of the various components, and the global power-law index-we froze the column densities of the warm absorber to the best-fit values obtained as we have already shown that the absorption does not vary on the timescales probed in this observation. Freezing these parameters was done in order to reduce computational time and could result in a slight underestimate of the true error. Nevertheless, the global power-law index is found to be $\Gamma=1.82 \pm 0.01$, very similar to that found for the time-averaged spectrum presented in Paper I. We investigated the possibility of a varying powerlaw index and show in Table 5 that gamma is perfectly consistent with the global value found above throughout the six periods. This supports the results presented in the previous section in which the difference-spectra below $10 \mathrm{keV}$ is shown to be well modeled with a simple power law.

The flux evolution of the three variable components is shown in Figure 13 (left). It is clear that as the $\left[10^{-3}, 10^{3}\right] \mathrm{keV}$ flux in the power-law continuum (blue) begins to decrease, the inner (red) reflection also decreases. However, in the latter half of the observation the flux in the power law decreases, whereas the inner reflection flux shows a gradual increase. The reflection arising from the outer part of the disk appears to be nearly constant. In fact, an equally good fit $\left(\chi^{2} / v=\right.$ $4514.6 / 3395(1.33)$; i.e., $\Delta \chi^{2}=16.9$ for 5 dof) can be achieved if we force it to be constant throughout all six periods. The evolution of these components manifests itself in the observed evolution of the reflection ratio $\mathcal{R}$, where we see in the right panels (top and middle) that the total contribution to the spectrum from the reflection component reaches a maximum in the second half of the observation and has a clear minimum during Period 2. We also show in the bottom-right panel the evolution of the inner ionization parameter $\left(\xi=L_{\text {int }} / n R^{2}\right)$. Assuming the intrinsic luminosity $\left(L_{\text {int }}\right)$ of the power-law continuum (as seen by the accretion disk) and the hydrogen number density of the disk $(n)$ remain constant, then the maximum change in ionization observed $\left(\xi_{\text {high }} / \xi_{\text {low }} \approx 4\right)$ implies an increase in the height of the corona (above the disk) by a factor of $\sim 2$ (assuming Newtonian geometry). Of course it is likely that there could be some contribution from both intrinsic variations in the continuum luminosity, and possibly changes in the hydrogen number density of the disk as well. In Figure 14 we visually depict the evolution of the three components during the course of the observation. The greater contribution from the inner reflection component (red) to the hard energy band during the last three periods in comparison to the first three is clearly seen (the near constancy of the reflection component arising from the outer parts of the accretion disk, shown in green, can be used as a reference point to highlight the level of change in the inner reflection flux). The extra flux at hard energies coming from the increase of the inner reflection component in comparison to the power-law continuum directly explains the presence of a hard excess in the difference-spectra presented in Section 3.4 for the second half of the observation in comparison to Period 2.

\section{DISCUSSION AND CONCLUSIONS}

The main results concerning the spectral variability of NGC 3783 as determined from the long Suzaku observation can be summarized as follows.

1. The spectral variability of NGC 3783 can be described by the superposition of a soft-variable component together with a quasi-constant hard component. The shape of this quasi-constant component (Figure 5) is hard in the 

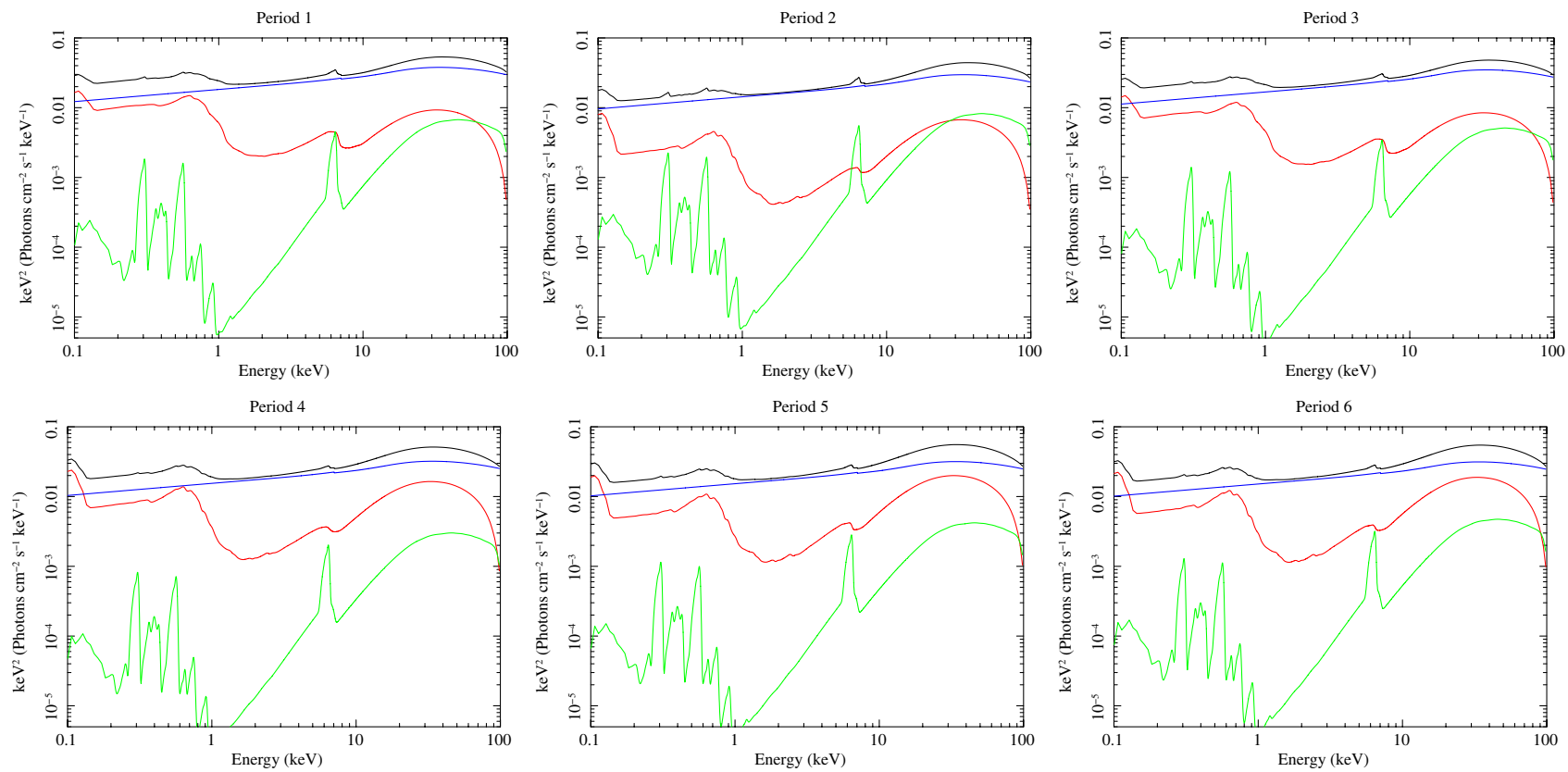

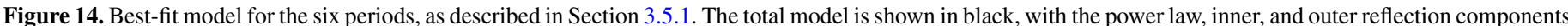
shown in blue, red, and green, respectively. The global blackbody, absorption, and narrow emission lines were removed for display.

(A color version of this figure is available in the online journal.)

3-10 keV range as found for several other AGNs, and flat both below $1 \mathrm{keV}$ and above $10 \mathrm{keV}$. These features are qualitatively similar to that expected from reflection arising from the inner parts of an accretion disk.

2. A simple power law provides a good fit to all the differencespectra between 3-10 keV suggesting that in this band the variability is dominated by variation in the normalization of the power-law-like Comptonized continuum.

3. Extending the power law to lower energies reveals the strong presence of a warm absorber (Figures 1, 8, and 11). The warm absorber does not vary with time or flux (see, e.g., Figure 7), which is consistent with previous studies of this source.

4. The presence of complex spectral evolution is clearly shown in Figure 10. The evolution of the softness ratio allows for the clear distinction of at least six "spectral periods" (Figure 2).

5. Time-resolved difference-spectra show the presence of an excess flux to a power-law continuum above $10 \mathrm{keV}$ in the latter half of the observation (Figure 11). This excess is not seen in the flux-resolved difference-spectra (Figure 8), suggesting that the further variable component above $10 \mathrm{keV}$ varies with time but not monotonically with flux.

6. Changes in the reflection fraction and possibly ionization state of the inner accretion disk could result in a reflection component having a quasi-constant level below $10 \mathrm{keV}$ but different contributions at higher energies. A model consisting of such a reflection component together with a power law and the associated Compton backscattered emission from cold material provides an excellent description of the continuum for the six distinct spectral periods seen in NGC 3783 (Figure 12).

Our time-domain spectral analysis presented in Section 3.5 presents a complex picture. To organize this discussion, we choose to focus on three primary observables, the observed power-law flux (PL), the ratio of the total disk reflection to the power law flux $\mathcal{R}=(\operatorname{Ref} 1+\operatorname{Ref} 2) / \mathrm{PL}$, and the ionization state of the inner accretion disk $(\xi)$. Thus, we have a threedimensional parameter space (PL, $\mathcal{R}, \xi$ ) that, broadly, describes the X-ray flux and spectral state of the central engine.

What are the theoretical expectations for the evolution of the source in this parameter space? We suppose for now that the observed continuum X-rays are emitted from a single structure ("the corona"), and that the observed reflection spectrum results from irradiation of the disk by that same structure. Within this picture, we can imagine at least three distinct causes for flux and spectral variability. First, suppose that the $\mathrm{X}$-ray emitting corona increases its luminosity while all other physical properties of the system (coronal geometry, coronal beaming, and disk density) remain constant. Clearly both the observed flux and the ionization parameter of the inner disk would increase in proportion, i.e., we would expect $\xi \propto$ PL. Since the geometry is unchanged, however, we would expect $\mathcal{R}$ to remain constant. The second type of variability corresponds to a change in the location, geometry, size, or beaming pattern of the corona while its luminosity and the disk properties remain unchanged or to changes in the geometry of the disk in a manner that some discrete Compton-thick clump of gas could move into the irradiation zone thus changing the (anisotropic) scattered/reflected emission directed into the line of sight. In the case that these changes lead to increased irradiation of the disk at the expense of flux reaching the observer (through, for example, increased gravitational light bending if the source moves closer to the black hole), PL would decrease while both $\xi$ and $\mathcal{R}$ would increase in rough proportion. The final type of change that we consider are density changes in the surface layers of the accretion disk (with all other aspects of the system remaining unchanged). Such behavior may result from viscous timescale evolution ${ }^{11}$ following some dramatic accretion or ejection event.

\footnotetext{
${ }^{11}$ For NGC 3783 the dynamical timescale $\left(\Omega^{-1}\right)$ at $6 r_{\mathrm{g}}$ is approximately $350 \mathrm{~s}$. Assuming $T_{\text {viscous }} \approx \alpha^{-1}(H / R)^{-2} \Omega^{-1}$ and $\alpha=(H / R)=0.1$ we have $T_{\text {viscous }} \sim 350 \mathrm{ks}$, similar to the duration of the observation.
} 

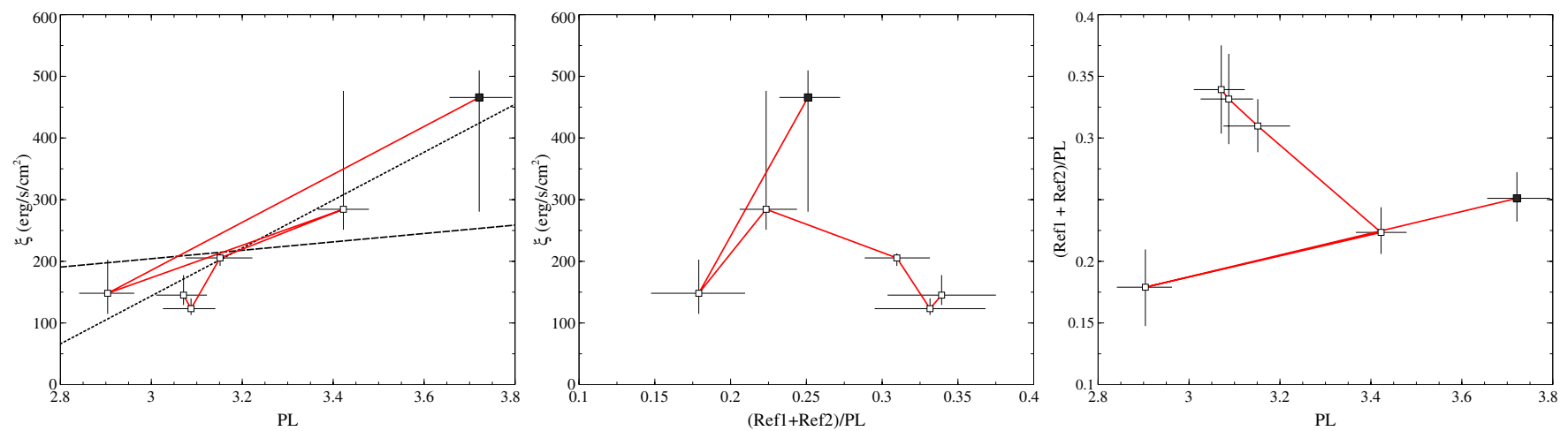

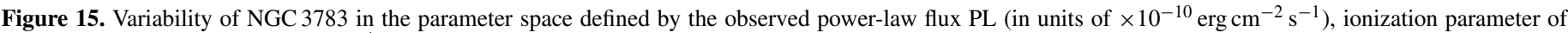

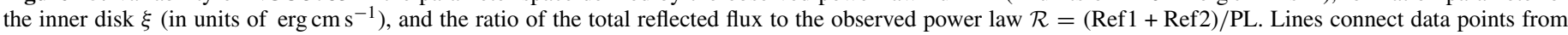

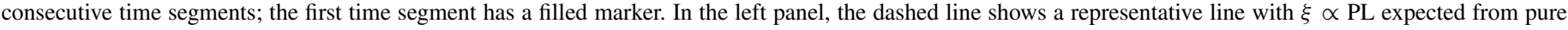

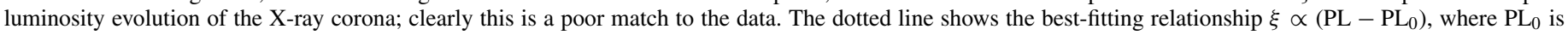
some constant flux component that has little or no associated reflection.

(A color version of this figure is available in the online journal.)

In this type of change, $\xi$ would respond inversely to density changes, while $\mathcal{R}$ and PL would remain unchanged.

Figure 15 shows the evolution of NGC 3783 projected onto the (PL, $\xi),(\mathrm{PL}, \mathcal{R})$, and $(\mathcal{R}, \xi)$ planes. Clearly, none of the three simple scenarios presented above can describe the complex behavior that we see-the variability must be due to a combination of physical changes. The fact that $\mathcal{R}$ changes demonstrates that there must be structural and/or geometrical changes in the $\mathrm{X}$-ray emitting corona and/or accretion disk. The most dramatic of these changes are in the final three data segments, although there is some weaker (and possibly flux-correlated) variability of $\mathcal{R}$ even between the first three data segments (Figure 15, right panel).

It is interesting to note that $\xi$ and PL do indeed seem correlated. However, we can only recover the linear proportionality between $\xi$ and PL (expected for "pure" coronal luminosity changes) if we assume that there is a constant flux component (corresponding to $\mathrm{PL}=2.63 \times 10^{-10} \mathrm{erg} \mathrm{cm}^{-2} \mathrm{~s}^{-1}$ ) that has no or very weak associated reflection spectrum, e.g., highly beamed emission from a steady jet. In this picture, all of the observed flux variability is due to the corona, which is also the principal source irradiating the disk. In addition to reproducing the observed $\xi-\mathrm{PL}$ correlations, it also explains the weak correlation of $\mathcal{R}$ with PL during the first three time segments-as PL increases, both its contribution and the contribution of its associated reflection spectrum increase relative to the constant component. The dramatic increase in $\mathcal{R}$ at the end of the data set still requires structural changes, however.

Like any data, the diagnostic power of these spectra are necessarily limited. However, a relatively simple and physically motivated analysis of the spectrum and source variability shows that reflection from a disk with corresponding changes in ionization provides an excellent description of the data. This discussion illustrates the power and utility of long X-ray data sets for examining AGN spectral variability. Such studies have the potential to yield new insights into the complex interplay between various physical processes in the cores of active galaxies.

R.C.R. would like to thank the Michigan Society of Fellows and NASA. R.C.R. is supported by NASA through the Einstein Fellowship Program, grant number PF1-120087. We are extremely grateful to our NASA and JAXA colleagues in the Suzaku project for enabling these Key Project data to be collected. This work was supported by NASA under the Suzaku Guest Observer grants NNX09AV43G and NNX10AR31G.

\section{REFERENCES}

Blustin, A. J., Branduardi-Raymont, G., Behar, E., et al. 2002, A\&A, 392, 453 Boldt, E. 1987, in IAU Symp. 124, Observational Cosmology, ed. A. Hewitt, G. Burbidge, \& L. Z. Fang (Cambridge: Cambridge Univ. Press), 611

Brenneman, L. W., Reynolds, C. S., Nowak, M. A., et al. 2011, ApJ, 736, 103

Dauser, T., Wilms, J., Reynolds, C. S., \& Brenneman, L. W. 2010, MNRAS, 409, 1534

Fabian, A. C., \& Vaughan, S. 2003, MNRAS, 340, L28

Fabian, A. C., Zoghbi, A., Ross, R. R., et al. 2009, Nature, 459, 540

Green, A. R., McHardy, I. M., \& Lehto, H. J. 1993, MNRAS, 265, 664

Halpern, J. P. 1984, ApJ, 281, 90

Kaspi, S., Brandt, W. N., George, I. M., et al. 2002, ApJ, 574, 643

Koyama, K., Tsunemi, H., Dotani, T., et al. 2007, PASJ, 59, 23

Krongold, Y., Nicastro, F., Brickhouse, N. S., Elvis, M., \& Mathur, S. 2005, ApJ, 622,842

Krongold, Y., Nicastro, F., Brickhouse, N. S., et al. 2003, ApJ, 597, 832

Laor, A. 1991, ApJ, 376, 90

Magdziarz, P., \& Zdziarski, A. A. 1995, MNRAS, 273, 837

McHardy, I. M. 1989, in Two Topics in X-Ray Astronomy, Volume 1: X Ray Binaries. Volume 2: AGN and the X Ray Background, ed. J. Hunt \&

B. Battrick (ESA Special Publication, Vol. 296; Noordwijk: ESA), 1111

Miller, J. M. 2007, ARA\&A, 45, 441

Miniutti, G., Fabian, A. C., Anabuki, N., et al. 2007, PASJ, 59, 315

Mitsuda, K., Bautz, M., Inoue, H., et al. 2007, PASJ, 59, 1

Nandra, K., George, I. M., Mushotzky, R. F., Turner, T. J., \& Yaqoob, T. 1997, ApJ, 477, 602

Nandra, K., O’Neill, P. M., George, I. M., \& Reeves, J. N. 2007, MNRAS, 382, 194

Netzer, H., Kaspi, S., Behar, E., et al. 2003, ApJ, 599, 933

Noda, H., Makishima, K., Yamada, S., et al. 2011, arXiv:1109.0457

Reynolds, C. S. 1997, MNRAS, 286, 513

Ross, R. R., \& Fabian, A. C. 2005, MNRAS, 358, 211

Shu, X. W., Yaqoob, T., \& Wang, J. X. 2010, ApJS, 187, 581

Takahashi, T., Abe, K., Endo, M., et al. 2007, PASJ, 59, 35

Taylor, R. D., Uttley, P., \& McHardy, I. M. 2003, MNRAS, 342, L31

Terashima, Y., Gallo, L. C., Inoue, H., et al. 2009, PASJ, 61S, 299

Uttley, P., Taylor, R. D., McHardy, I. M., et al. 2004, MNRAS, 347, 1345

Vaughan, S., \& Fabian, A. C. 2004, MNRAS, 348, 1415

Vaughan, S., Fabian, A. C., \& Nandra, K. 2003, MNRAS, 339, 1237

Vestergaard, M., \& Peterson, B. M. 2006, ApJ, 641, 689

Walton, D. J., Reis, R. C., \& Fabian, A. C. 2010, MNRAS, 408, 601

Zdziarski, A. A., Lubiński, P., Gilfanov, M., \& Revnivtsev, M. 2003, MNRAS, 342,355

Zoghbi, A., Fabian, A. C., Uttley, P., et al. 2010, MNRAS, 401, 2419 